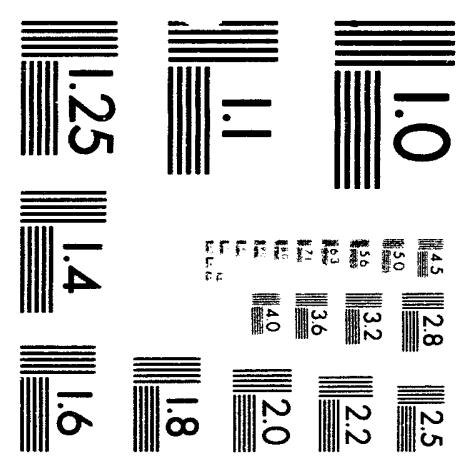



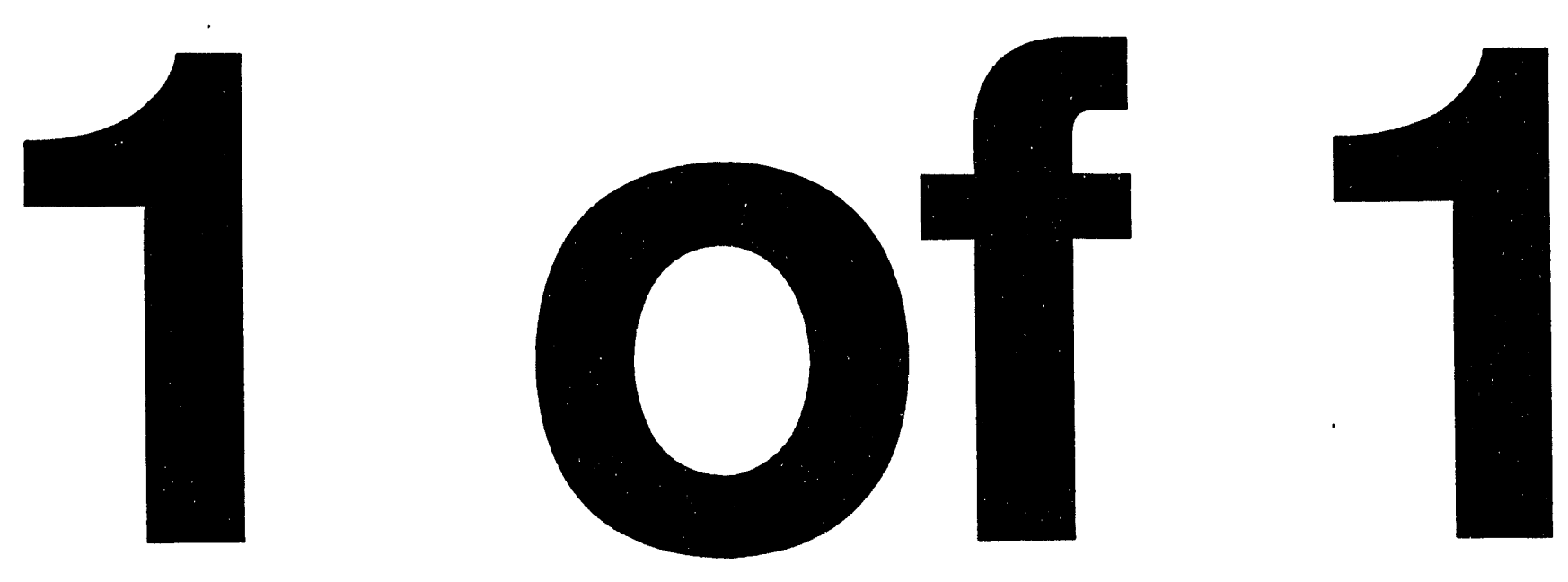


\section{K-AREA AND PAR POND SEWAGE SLUDGE APPLICATION SITES GROUNDWATER MONITORING REPORT (U)}

PUBLICATION DATE: OCTOBER 1993

Authorized Derivative Classifier:

Thichel Bullington

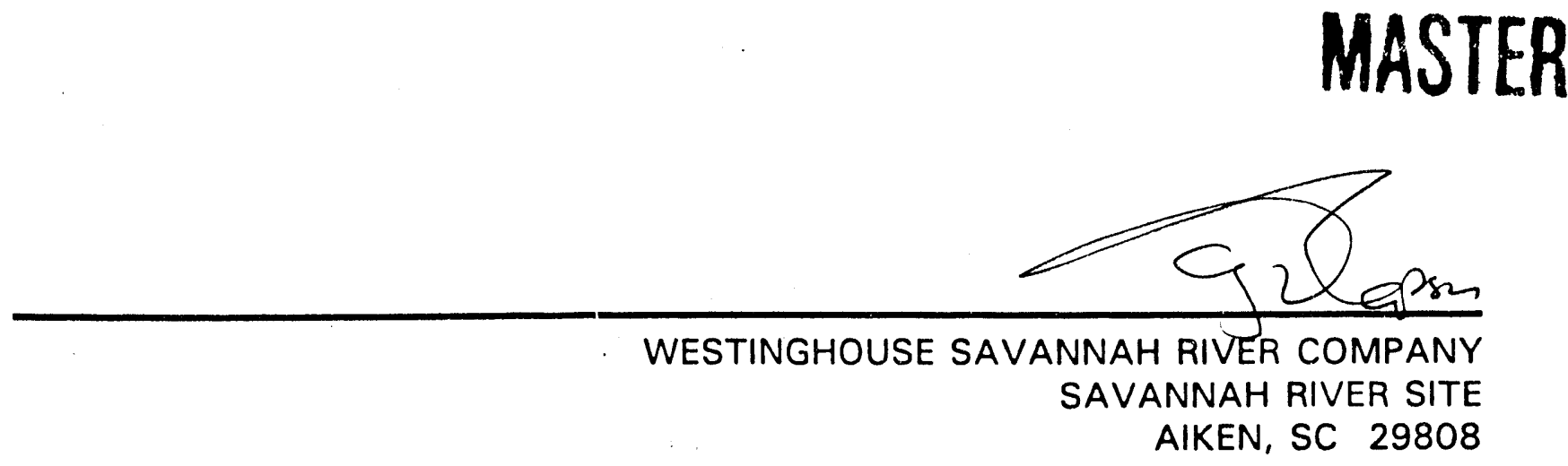

Prepared for the U.S. Department of Energy under Control Cnntract No. DE-AC09-89SR18035 


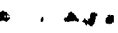




\section{Abstract}

Samples from the three wells at the K-Area Sewage Sludge Application Site (KSS wells) and the three wells at the Par Pond Sewage Sludge Application Site (PSS wells) are analyzed quarterly for constituents required by South Carolina Department of Health and Environmental Control Construction Permit 13,173 and, as requested, for other constituents as part of the Savannah River Site Groundwater Monitoring Program. Annual analyses for other constituents, primarily metals, also are required by the permit.

During second quarter 1993, no permit-required constituent exceeded standards at the two sites. Iron and aluminum exceeded standards during first quarter 1993. Elevated levels of aluminum and iron at these two sites may be concurrent with a change in analytical methodology. Neither of these constituents was analyzed second quarter 1993. 
WSRC-TR-93-398 


\section{Contents}

Page

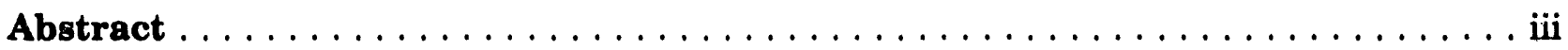

List of Figures $\ldots \ldots \ldots \ldots \ldots \ldots \ldots \ldots \ldots \ldots \ldots \ldots \ldots \ldots \ldots \ldots \ldots \ldots$

List of Tables $\ldots \ldots \ldots \ldots \ldots \ldots \ldots \ldots \ldots \ldots \ldots \ldots \ldots \ldots \ldots \ldots \ldots \ldots$ viii

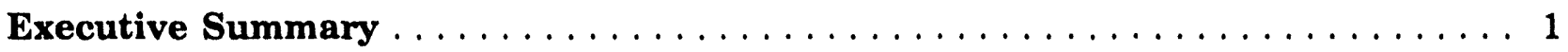

Introduction $\ldots \ldots \ldots \ldots \ldots \ldots \ldots \ldots \ldots \ldots \ldots \ldots \ldots \ldots$

Discussion $\ldots \ldots \ldots \ldots \ldots \ldots \ldots \ldots \ldots \ldots \ldots \ldots \ldots \ldots \ldots \ldots$

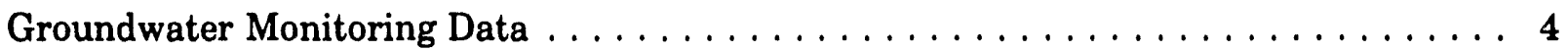

Analytical Results Exceeding Standards $\ldots \ldots \ldots \ldots \ldots \ldots \ldots \ldots \ldots \ldots$

Water Elevations and Groundwater Flow Directions $\ldots \ldots \ldots \ldots \ldots \ldots \ldots \ldots 6$

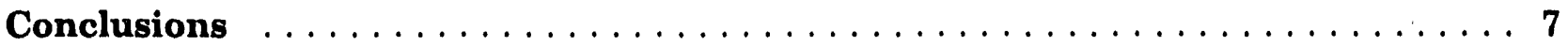

References Cited $\ldots \ldots \ldots \ldots \ldots \ldots \ldots \ldots \ldots \ldots \ldots \ldots \ldots$

Errata $\ldots \ldots \ldots \ldots \ldots \ldots \ldots \ldots \ldots \ldots \ldots \ldots \ldots \ldots$

Appendix A-Final Primary Drinking Water Standards $\ldots \ldots \ldots \ldots \ldots$ A-1

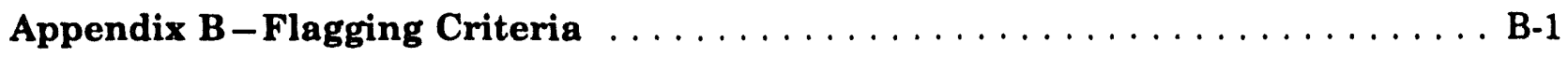

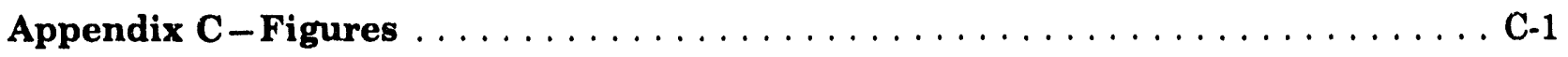

Appendix D-Groundwater Monitoring Results Tables $\ldots \ldots \ldots \ldots \ldots \ldots$ D-1

Appendix E-Data Quality/Useability Assessment $\ldots \ldots \ldots \ldots \ldots \ldots \ldots$ E-1 
WSRC-TR-93-398 


\section{List of Figures}

Page

1. Location of the K-Area and Par Pond Sewage Sludge Application Sites at the Savannah River Site

2. Location of Groundwater Monitoring Wells at the K-Area Sewage Sludge Application Site

3. Piezometric Map of the Water Table at the K-Area Sewage Sludge Application Site

4. Location of Groundwater Monitoring Wells at the Par Pond Sewage Sludge Application Site C-5

5. Water Table Elevations at the Par Pond Sewage Sludge Application Site . . . . . C C-6 


\section{List of Tables}

Page

1. Maximum Levels of Constituents Exceeding the Final Primary Drinking Water Standards at the K-Area Sewage Sludge Application Site $\ldots \ldots \ldots \ldots \ldots \ldots$ D-5

2. Maximum Levels of Constituents Exceeding Half the Final Primary Drinking Water Standards or Other Flag 1 or Flag 2 Criteria at the K-Area Sewage

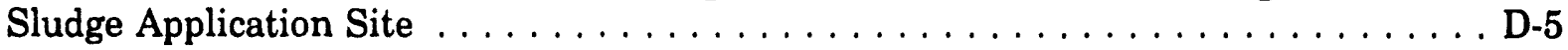

3. Maximum Levels of Constituents Exceeding the Final Primary Drinking Water Standards at the Par Pond Sewage Sludge Application Site . . . . . . . . . . . D-6

4. Maximum Levels of Constituents Exceeding Half the Final Primary Drinking Water Standards or Other Flag 1 or Flag 2 Criteria at the Par Pond Sewage

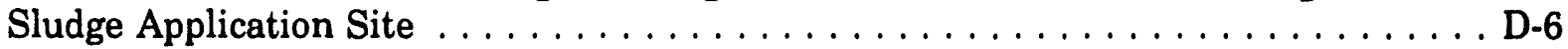

5. Groundwater Monitoring Results for Individual Wells at the K-Area Sewage

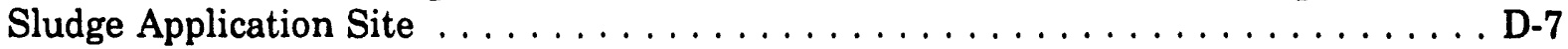

6. Groundwater Monitoring Results for Individual Wells at the Par Pond Sewage

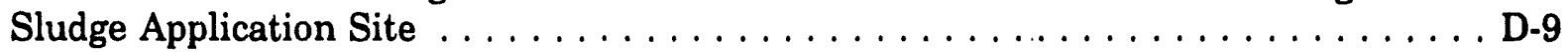




\section{Executive Summary}

During second quarter 1993, samples from the three monitoring wells at the K-Area site (KSS series) and the three monitoring wells at the Par Pond site (PSS series) were analyzed for constituents required by South Carolina Department of Health and Environmental Control Construction Permit 13,173 and for other constituents as part of the Savannah River Site (SRS) Groundwater Monitoring Program. This report describes monitoring results that exceeded the final Primary Drinking Water Standards (PDWS) or the SRS flagging criteria.

During second quarter 1993, no constituents exceeded the final PDWS or any other flagging criteria at the K-Area and Par Pond Sewage Sludge Application Sites. During first quarter 1993, aluminum and iron exceeded the SRS Flag 2 criteria in one or more of the KSS and the PSS wells. These constituents were not analyzed second quarter 1993. In the KSS well series, the field measurement for alkalinity ranged as high as $35 \mathrm{mg} / \mathrm{L}$ in well KSS $1 \mathrm{D}$. Alkalinity measurements were zero in the PSS wells, except for a single measurement of $1 \mathrm{mg} / \mathrm{L}$ in well PSS $1 \mathrm{D}$.

Historical and current water-level elevations at the K-Area Sewage Sludge Application Site indicate that the groundwater flow direction is south to southwest (SRS grid coordinates). The groundwater flow direction at the Par Pond Sewage Sludge Application Site could not be determined second quarter 1993. 
WSRC-TR-93-398 


\section{Introduction}

The sewage sludge application sites at the Savannah River Site (SRS) were originally the subject of a research program, begun in 1980, using domestic sewage sludge to reclaim borrow pits and to enhance forest productivity at SRS. After sludge was applied to the sites, hardwoods and pines were planted to quantify the wood biomass that could be produced using the sludge as a fertilizer and soil conditioner.

The 17-acre K-Area Sewage Sludge Application Site lies southeast of K Area on the west bank of Pen Branch (Figures 1 and 2, Appendix C). The Par Pond Sewage Sludge Application Site includes 22 acres south of Par Pond (Figures 1 and 4, Appendix C). As part of the research program, wastewater sludge was applied to the K-Area and Par Pond sites in 1981 under Industrial Waste Permit 175. These sites received a second application of sludge in 1988. This last application, from the Central Shops Sanitary Sludge Lagoon, was a one-time occurrence in accordance with Construction Permit 13,173, issued in April 1987 by the South Carolina Department of Health and Environmental Control (SCDHEC). Both sites are now inactive.

In 1988, SRS determined that new monitoring wells were required at the K-Area and Par Pond Sewage Sludge Application Sites to assess the effects of sewage sludge application on groundwater quality. SRS installed three wells at the K-Area site and three wells at the Par Pond site after approval by SCDHEC. These wells, which were first sampled during fourth quarter 1988, are designated KSS 1D, 2D, and 3D and PSS 1D, 2D, and 3D. All wells monitor the water table and are constructed of 4-in. polyvinyl chloride pipe.

In 1989, the K-Area and Par Pond Sewage Sludge Application Sites became part of the Resource Conservation and Recovery Act Facilities Investigation/Remedial Investigation program because chlordane was found in the sludge from the Central Shops lagoon. Chlordane has not appeared above detection limits in any KSS or PSS well since at least first quarter 1991.

The SRS Environmental Protection Department/Environmental Monitoring Section (EPD/EMS) samples the KSS and PSS wells each quarter, and the SRS Environmental Restoration Department reports the results of this sampling to SCDHEC as required by Special Condition 4 of Construction Permit 13,173. 


\section{Discussion}

\section{Groundiwater Monitoring Data}

The EPD/EMS sampling procedure (WSRC, 1992) requires evacuation of a minimum of two well volumes and stabilization of $\mathrm{pH}$, specific conductance, and turbidity prior to sample collection. Stability is established when a minimum of three successive measurements of each of these parameters, taken within a given time period, is within a specified tolerance range.

If a well pumps dry before two well volumes are purged or before stabilization is achieved, it must be revisited within 24 hours for the data to be considered from a single sampling event. On the second visit within 24 hours, samples are taken without further purging or stability measurements; thus, these samples may not be representative of groundwater quality. Tables 5 and 6 (Appendix D) list the number of well volumes purged from the KSS and PSS wells, respectively, during second quarter 1993. Wells KSS 1D and PSS 3D went dry during purging; both recovered sufficiently to sample within 24 hours. Well PSS 3D has consistently purged dry since its installation. At present, all KSS and PSS wells have single-speed centrifugal pumps.

Samples from the monitoring wells at the K-Area and Par Pond Sewage Sludge Application Sites are analyzed for the following parameters as required by SCDHEC Construction Permit 13,173:

- Quarterly analyses for specific conductance and $\mathrm{pH}$ (laboratory measurements)

- Quarterly analyses for water quality indicators: chloride, nitrate, nitrite, sodium, and total dissolved solids

- Annual analyses for cadmium, calcium, copper, iron, lead, magnesium, manganese, nickel, potassium, and total phosphates (as phosphorus)

The KSS and PSS wells may also receive additional analyses for other constituents, including chlordane, as part of the SRS Groundwater Monitoring Program.

Monitoring results that equaled or exceeded the Safe Drinking Water Act final Primary Drinking Water Standards (PDWS) or drinking water screening levels, as established by the U.S. Environmental Protection Agency (EPA) (Appendix A); the South Carolina final PDWS for lead (Appendix A); or SRS flagging criteria based on final and proposed PDWS, Secondary Drinking Water Standards, and method detection limits (Appendix B) are described in this report. Constituent levels that equaled or exceeded the final PDWS or SRS Flag 2 criteria are described as elevated or as exceeding or above standards. 
The final PDWS for individual analytes given in Appendix A may not always match the SRS flagging criteria in Appendix B. The final PDWS are used as guidelines in this compliance report to meet regulatory requirements; the flagging criteria are used by EPD/EMS to identify relative levels of constituents in the groundwater and as guides for scheduling groundwater sampling.

Appendix C provides the locations of the monitored sites at SRS (Figure 1), the individual KSS and PSS monitoring wells (Figures 2 and 4, respectively), and the groundwater flow directions beneath the sites (Figures 3 and 5). Monitoring results tables and analyses that exceeded EPA-approved holding times are in Appendix D, and information on the assessment of data quality and useability is in Appendix E.

\section{Analytical Results Exceeding Standards}

Tables 1 and 3 (Appendix D) summarize constituents that exceeded the final PDWS (see Appendix A) at the K-Area Sewage Sludge Application Site or the Par Pond Sewage Sludge Application Site, respectively, during second quarter 1993. None of the constituents analyzed exceeded the final PDWS in the KSS or PSS wells.

Constituents exceeding other flagging criteria (see Appendix B) during second quarter 1993 are summarized in Table 2 (Appendix D) for the KSS wells and Table 4 (Appendix D) for the PSS wells. None of the constituents analyzed exceeded the other flagging criteria in the KSS or PSS well series during second quarter 1993. During first quarter 1993, upgradient well KSS 1D contained elevated aluminum and iron concentrations. These constituents were not analyzed during second quarter 1993. Well KSS 2D, downgradient within the southern border of the application area, exhibited an elevated concentration of aluminum first quarter 1993; this constituent was not analyzed second quarter 1993. Downgradient well KSS 3D contained no elevated constituents either quarter.

Upgradient well PSS 1D and downgradient wells PSS 2D and PSS 3D contained no elevated constituents during second quarter 1993. During first quarter 1993, PSS 1D and PSS 3D both contained elevated concentrations of aluminum and iron. These constituents were not analyzed second quarter 1993. See the Conclusions section of this report for a discussion of the history of these constituents.

Results are presented for all analyzed constituents for the KSS and PSS wells in Tables 5 and 6 , respectively (Appendix D). Chlordane was not analyzed in any of the three KSS or the three PSS wells during the quarter.

Presently, SRS sets no flagging criteria for alkalinity. In the KSS well series, alkalinity ranged as high as $35 \mathrm{mg} / \mathrm{L}$ in well $\mathrm{KSS} 1 \mathrm{D}$. Alkalinity measurements were zero in the PSS wells, except for a reading of $1 \mathrm{mg} / \mathrm{L}$ in well PSS $1 \mathrm{D}$. 


\section{Water Elevations and Groundwater Flow Directions}

Water-level elevations at the K-Area Sewage Sludge Application Site indicate that the groundwater flow direction is generally south to southwest (SRS coordinates) (Figure 3, Appendix C), although the nearly linear orientation of these wells makes such a determination tentative. The groundwater flow directions historically have been south to southwest (SRS coordinates).

During second quarter 1993, only two water-level elevations were obtained at the Par Pond Sewage Sludge Application Site. At the time of sampling, there was no water in the standpipe of well PSS 3D. Thus, determination of flow direction during this quarter was not possible. Historically, flow direction has been reported as south to southwest (SRS coordinates) (Figure 5, Appendix C). 


\section{Conclusions}

- During second quarter 1993, as in all quarters of 1992 and the first quarter of 1993, no constituents exceeded the fi..al PDWS at the K-Area and Par Pond Sewage Sludge Application Sites.

- During the first quarter of 1993, aluminum was elevated in wells KSS 1D and 2D and PSS 1D and 3D. Iron was elevated in wells KSS 1D and PSS 1D and 3D. Aluminum was not analyzed in groundwater samples from KSS or PSS wells during 1992. Iron was analyzed only during first quarter in KSS wells and only during first and third quarters 1992 in PSS wells; elevated iron concentrations were infrequent. The occurrence of elevated iron during first quarter 1993 may reflect the change to analyses of unfiltered metals samples. These constituents were not analyzed second quarter 1993. Aluminum is part of comprehensive analyses, which occur once every other year and are not required by the permit. Iron is requested in the permit for annual analysis only.

- Chlordane was not analyzed in the three KSS and the three PSS wells during second quarter 1993. This constituent was requested by Kelly Ward for first quarter 1993 only. Chlordane was analyzed third quarter 1991, fourth quarter 1991, first quarter 1992, third quarter 1992, fourth quarter 1992, and first quarter 1993 in some or all of the KSS and PSS wells. Chlordane was not detected in any of these analyses.

- Groundwater flow direction at the K-Area Sewage Sludge Application Site was generally south to southwest (SRS grid coordinates) (Figure 3, Appendix C); groundwater flow direction at the Par Pond Sewage Sludge Application Site could not be determined during second quarter 1993, but historically it has been toward the south to southwest (Figure 5, Appendix C).

- In the KSS well series, alkalinity ranged as high as $35 \mathrm{mg} / \mathrm{L}$ in well KSS 1D. Alkalinity measurements were zero in the PSS wells, except for a reading of $1 \mathrm{mg} / \mathrm{L}$ in PSS 1D.

- Wells KSS 1D and PSS 3D went dry during purging; thus, the samples from these wells may not be representative groundwater samples. 


\section{References Cited}

WSRC (Westinghouse Savannah River Company), 1992. Hydrogeologic Data Collection Procedures and Specifications: Sampling Groundwater Monitoring Wells. Manual 3Q5, Chapter 15, Rev. 1. Environmental Protection Department, Environmental Monitoring Section, Savannah River Site, Aiken, SC. 


\section{Errata}

First through Third Quarters 1992:

- Chlordane analysis was requested as part of the Base/Neutral/Acid suite of analyses as described in the Environmental Protection Department/Environmental Monitoring Section contract with the analytical laboratory. However, Roy F. Weston, Inc., which conducted the analyses for first through third quarters 1992, does not include chlordane in its Base/Neutral/Acid suite of analyses. Chlordane analysis was conducted by General Engineering Laboratories for fourth quarter 1992.

First through Fourth Quarters 1992:

- Some results for earlier quarters of 1992 that are presented in the results tables of the fourth quarter 1992 report may differ from the results presented in the earlier reports, and reported results may not match reported sample dates. These differences arise from the following: (1) the computer program that creates the results tables was revised beginning second quarter 1992 to present the highest value for analytes with more than one result (previously, the program presented the first value encountered in the database); (2) a new computer program, which rounds numbers differently from the former computer program, was first used during third quarter 1992; and (3) some reanalyses may have been performed by the laboratories after the quarterly reports had gone to press. The sample dates in the tables are the dates when the field data were collected. These dates may differ from the dates of the laboratory analyses if the highest results were obtained for samples collected on different dates. 
WSRC-TR-93-398 
WSRC-TR-93-398

\section{Appendix A - Final Primary Drinking Water Standards}


Final Primary Drinking Water Standards

\begin{tabular}{|c|c|c|c|c|}
\hline Analvte & Unit & Level & Status & Source \\
\hline Antimony & $\mu g / L$ & 6 & Final & EPA, 1992b \\
\hline Arsenic & $\mu g / L$ & 50 & Final & EPA, $1992 a$ \\
\hline Asbestos & fibers/L & $7,000,000$ & Final & EPA, 1992a \\
\hline Barium & $\mu g / L$ & 2,000 & Final & EPA, 1992a \\
\hline Benzene & $\mu g / L$ & 5 & Final & EPA, 1992a \\
\hline Benzolalpyrene & $\mu g / L$ & 0.2 & Final & EPA, 1992b \\
\hline Beryllium & $\mu_{g} / L$ & 4 & Final & EPA, 1992b \\
\hline Bis(2-ethylhexyl) phthalate & $\mu g / L$ & 6 & Final & EPA, $1992 b$ \\
\hline Bromodichloromethane & $\mu g / L$ & $100^{8}$ & Final & EPA, 1992a \\
\hline Bromoform & $\mu g / L$ & $100^{8}$ & Final & EPA, $1992 a$ \\
\hline 2-sec-Butyl-4,6-dinitrophenol & $\mu g / L$ & 7 & Final & EPA, 1992b \\
\hline Cadmium & $\mu g / L$ & 5 & Final & EPA, 1992a \\
\hline Carbon tetrachloride & $\mu g / L$ & 5 & Final & EPA, 1992a \\
\hline Chlordane & $\mu g / L$ & 2 & Final & EPA, 1992a \\
\hline Chlorobenzene & $\mu g / L$ & 100 & Final & EPA, 1992a \\
\hline Chloroethene (Vinyl chloride) & $\mu g / L$ & 2 & Final & EPA, 1992a \\
\hline Chloroform & $\mu g / L$ & $100^{\theta}$ & Final & EPA, 1992a \\
\hline Chromium & $\mu g / L$ & 100 & Final & EPA, 1992a \\
\hline Copper & $\mu g / L$ & 1,300 & Final & EPA, 1992a \\
\hline Cyanide & $\mu g / L$ & 200 & Final & EPA, 1992b \\
\hline Dibromochloromethane & $\mu g / L$ & $100^{a}$ & Final & EPA, 1992a \\
\hline Dibromochloropropane & $\mu g / L$ & 0.2 & Final & EPA, 1992a \\
\hline 1,2-Dibromoethane (Ethylene dibromide) & $\mu g / L$ & 0.05 & Final & EPA, 1992a \\
\hline 1,2-Dichlorobenzene & $\mu g / L$ & 600 & Final & EPA, 1992a \\
\hline 1,4-Dichlorobenzene & $\mu g / L$ & 75 & Final & EPA, 1992a \\
\hline 1,2-Dichloroethane & $\mu g / L$ & 5 & Final & EPA, 1992a \\
\hline 1,1-Dichloroethene & $\mu g / L$ & 7 & Final & EPA, $1992 a$ \\
\hline 1,2-Dichloroethene & $\mu g / L$ & 50 & Final & EPA, $1992 b$ \\
\hline cis-1,2-Dichloroethene & $\mu g / L$ & 70 & Final & EPA, 1992a \\
\hline trans-1,2-Dichloroethene & $\mu g / L$ & 100 & Final & EPA, 1992a \\
\hline Dichloromethane (Methylene chloride) & $\mu g / L$ & 5 & Final & EPA, 1992b \\
\hline 2,4-Dichlorophenoxyacetic acid & $\mu g / L$ & 70 & Final & EPA, $1992 a$ \\
\hline 1,2-Dichloropropane & $\mu g / L$ & 5 & Final & EPA, 1992a \\
\hline Endrin & $\mu g / L$ & 2 & Final & EPA, 1992b \\
\hline Ethylbenzene & $\mu g / L$ & 700 & Final & EPA, 1992a \\
\hline Fluoride & $\mu g / L$ & 4,000 & Final & EPA, $1992 a$ \\
\hline Gross alphab & $\mathrm{pCi} / \mathrm{L}$ & $1.5 E+01$ & Final & EPA, 1992a \\
\hline Heptachlor & $\mu g / L$ & 0.4 & Final & EPA, 1992a \\
\hline Heptachlor epoxide & $\mu g / L$ & 0.2 & Final & EPA, 1992a \\
\hline Hexachlorobenzene & $\mu g / L$ & 1 & Final & EPA, 1992b \\
\hline Hexachlorocyclopentadiene & $\mu g / L$ & 50 & Final & EPA, 1992b \\
\hline Lead & $\mu g / L$ & 50 & Final & SCDHEC, 198 \\
\hline Lindane & $\mu g / L$ & 0.2 & Final & EPA, 1992a \\
\hline Mercury & $\mu g / L$ & 2 & Final & EPA, 1992a \\
\hline Methoxychlor & $\mu g / L$ & 40 & Final & EPA, 1992a \\
\hline Nickel & $\mu g / L$ & 100 & Final & EPA, 1992b \\
\hline Nitrate as nitrogen & $\mu g / L$ & 10,000 & Final & EPA, 1992a \\
\hline Nitrate-nitrite as nitrogen & $\mu g / L$ & 10,000 & Final & EPA, 1992a \\
\hline Nitrite as nitrogen & $\mu \mathrm{g} / \mathrm{L}$ & 1,000 & Final & EPA, 1992a \\
\hline Nonvolatile beta ${ }^{c}$ & $\mathrm{pCi} / \mathrm{L}$ & $5 E+01$ & Final & EPA, 1977 \\
\hline PCBs ${ }^{d}$ & $\mu \mathrm{g} / \mathrm{L}$ & 0.5 & Final & EPA, 1992a \\
\hline Pentachlorophenol & $\mu g / L$ & 1 & Final & EPA, 1992a \\
\hline Radium, total (Radium-226 and -228 ) & $\mathrm{pCi} / \mathrm{L}$ & $5 E+00$ & Final & EPA, 1992a \\
\hline
\end{tabular}


Analyte

Selenium

Strontium-89/90

Strontium-90

Styrene

2,3,7,8-TCDD

Tetrachloroethylene

Thallium

Toluene

Total trihalomethanes

Toxaphene

2,4,5-TP (Silvex)

1,2,4-Trichlorobenzene

1,1,1-Trichloroethane

1,1,2-Trichloroethane

Trichloroethylene

Tritium

Xylenes

$\begin{array}{llll}\text { Unit } & \text { Level } & \text { Status } & \text { Source } \\ \mu g / L & 50 & \text { Final } & \text { EPA, 1992a } \\ \mathrm{pCi} / \mathrm{L} & 8 \mathrm{E}+00 & \text { Final } & \text { EPA, 1992a } \\ \mathrm{pCi} / \mathrm{L} & 8 \mathrm{E}+00 & \text { Final } & \text { EPA, 1992a } \\ \mu \mathrm{g} / \mathrm{L} & 100 & \text { Final } & \text { EPA, 1992a } \\ \mu \mathrm{g} / \mathrm{L} & 0.00003 & \text { Final } & \text { EPA, 1992b } \\ \mu \mathrm{g} / \mathrm{L} & 5 & \text { Final } & \text { EPA, 1992a } \\ \mu \mathrm{g} / \mathrm{L} & 2 & \text { Final } & \text { EPA, 1992b } \\ \mu \mathrm{g} / \mathrm{L} & 1,000 & \text { Final } & \text { EPA, 1992a } \\ \mu \mathrm{g} / \mathrm{L} & 100 & \text { Final } & \text { EPA, 1992a } \\ \mu \mathrm{g} / \mathrm{L} & 3 & \text { Final } & \text { EPA, 1992a } \\ \mu \mathrm{g} / \mathrm{L} & 50 & \text { Final } & \text { EPA, 1992a } \\ \mu \mathrm{g} / \mathrm{L} & 70 & \text { Final } & \text { EPA, 1992b } \\ \mu \mathrm{g} / \mathrm{L} & 200 & \text { Final } & \text { EPA, 1992a } \\ \mu \mathrm{g} / \mathrm{L} & 5 & \text { Final } & \text { EPA, 1952b } \\ \mu \mathrm{g} / \mathrm{L} & 5 & \text { Final } & \text { EPA, 1992a } \\ \mathrm{pCi} / \mathrm{mL} & 2 \mathrm{E}+01 & \text { Final } & \text { EPA, 1992a } \\ \mu \mathrm{g} / \mathrm{L} & 10,000 & \text { Final } & \text { EPA, 1992a }\end{array}$

a This value is the drinking water standard for total trihalomethanes (the sum of bromoform, bromodichloromethane, chloroform, and dibromochloromethane).

b The standard given is for gross alpha including radium-226 but excluding radon and uranium.

c This is the screening level above which providers of public drinking water should perform analyses for specific man-made radionuclides. The standard for the total dose equivalent from all such radionuclides is 4 mrem per year.

d Analyses were conducted in 1992 for the following: PCB 1016, PCB 1221, PCB 1232, PCB 1242, PCB 1248, PCB 1254, and PCB 1260.

- For double radionuclide analyses where each separate radionuclide has its own standard, the more stringent standard is used.

\section{References}

EPA (U.S. Environmental Protection Agency), 1977. National Interim Primary Drinking Water Regulations, EPA570/9-76-003. Washington, DC.

EPA (U.S. Environmental Protection Agency), 1992a. National Primary Drinking Water Regulations, Code of Federal Regulations, Title 40, Part 141, pp. 589-729. Washington, DC.

EPA (U.S. Environmental Protection Agencyl, 1992b. National Primary Drinking Water Regulations-Synthetic Organic Chemicals and Inorganic Chemicals; National Primary Drinking Water Regulations Implementation. Federal Register, July 17, 1992, pp. 31776-31849. Washington, DC.

SCDHEC (South Carolina Department of Health and Environmental Control), 1981. State Primary Drinking Water Regulations, R.61-58.5. Columbia, SC. 
WSRC-TR-93-398 
Appendix B - Flagging Criteria 


\section{Flagging Criteria}

The Savannah River Site Environmental Protection Department/Environmental Monitoring Section (EPD/EMS) flagging criteria are as follows:

- Flag 2 criteria for constituents equal the Safe Drinking Water Act (SDWA) final Primary Drinking Water Standard (PDWS), the SDWA proposed PDWS, or the SDWA Secondary Drinking Water Standard (SDWS). If a constituent does not have a drinking water standard, the Flag 2 criterion equals 10 times the method detection limit (MDL) calculated as the 90 th percentile detection limit obtained recently by one of the primary analytical laboratories.

- Flag 1 criteria for constituents equal one-half of the final PDWS, one-half the proposed PDWS, or one-half the SDWS. If a constituent does not have a drinking water standard, the Flag 1 criterion equals 5 times the MDL calculated as the 90th percentile detection limit obtained recently by one of the primary analytical laboratories.

- Flag 0 criteria are assigned to constituent levels below Flag 1 criteria, constituent levels below the sample detection limits, or constituents having no flagging criteria.

The following parameters are not assigned flagging criteria: alkalinity, calcium, color, corrosivity, Eh, magnesium, odor, potassium, silica, sodium, total dissolved solids, total phosphates (as P), total phosphorus, and turbidity. In addition, common laboratory contaminants and cleaners including some phthalates, ketones, and toluene are not assigned flagging criteria.

Analyte

Acenaphthene

Acenaphthylene

Acetone

Acetonitrile (Methyl cyanide)

Acetophenone

2-Acetylaminofluorene

Acrolein

Acrylonitrile

Actinium-228

Aldrin

Alkalinity (as $\mathrm{CaCO}_{3}$ )

Allyl chloride

Aluminum

Americium-241

Americium-243

4-Aminobiphenyl

Ammonia

Ammonia nitrogen

Aniline

Anthracene

Antimony

Antimony- 125

Aramite

Arsenic

\begin{tabular}{|c|c|c|}
\hline Unit & Flag 1 & Flag 2 \\
\hline$\mu g / L$ & 50 & 100 \\
\hline$\mu g / L$ & 50 & 100 \\
\hline$\mu g / L$ & 500 & 1,000 \\
\hline$\mu g / L$ & 500 & 1,000 \\
\hline$\mu g / L$ & 50 & 100 \\
\hline$\mu g / L$ & 50 & 100 \\
\hline$\mu g / L$ & 100 & 200 \\
\hline$\mu g / L$ & 100 & 200 \\
\hline $\mathrm{pCi} / \mathrm{L}$ & $1.64 E+03$ & $3.27 E+03$ \\
\hline$\mu g / L$ & 0.25 & 0.5 \\
\hline & No flag & No flag \\
\hline$\mu g / L$ & 250 & 500 \\
\hline$\mu g / L$ & 25 & 50 \\
\hline $\mathrm{pCi} / \mathrm{L}$ & $3.17 E+00$ & $6.34 E+00$ \\
\hline $\mathrm{pCi} / \mathrm{L}$ & $3.19 E+00$ & $6.37 E+00$ \\
\hline$\mu g / L$ & 50 & 100 \\
\hline$\mu g / L$ & 500 & 1,000 \\
\hline$\mu g / L$ & 500 & 1,000 \\
\hline$\mu \mathrm{g} / \mathrm{L}$ & 50 & 100 \\
\hline$\mu \mathrm{g} / \mathrm{L}$ & 50 & 100 \\
\hline$\mu g / L$ & 3 & 6 \\
\hline $\mathrm{pCi} / \mathrm{L}$ & $1.5 E+02$ & $3 E+02$ \\
\hline$\mu g / L$ & 50 & 100 \\
\hline$\mu g / L$ & 25 & 50 \\
\hline
\end{tabular}

Source $^{\mathbf{a}}$

EPA Method 8270

EPA Method 8270

EPA Method 8240

EPA Method 8240

EPA Method 8270

EPA Method 8270

EPA Method 8240

EPA Method 8240

Proposed PDWS (EPA, 1991)

EPA Method 8080

Set by EPD/EMS

EPA Method 8240

SDWS (EPA, 1992c)

Proposed PDWS (EPA, 1991)

Proposed PDWS (EPA, 1991)

EPA Method 8270

APHA Method 417B

EPA Method 350.1

EPA Method 8270

EPA Method 8270

Final PDWS (EPA, 1992b)

Final PDWS (EPA, 1977)

EPA Method 8270

Final PDWS (EPA, 1992a) 


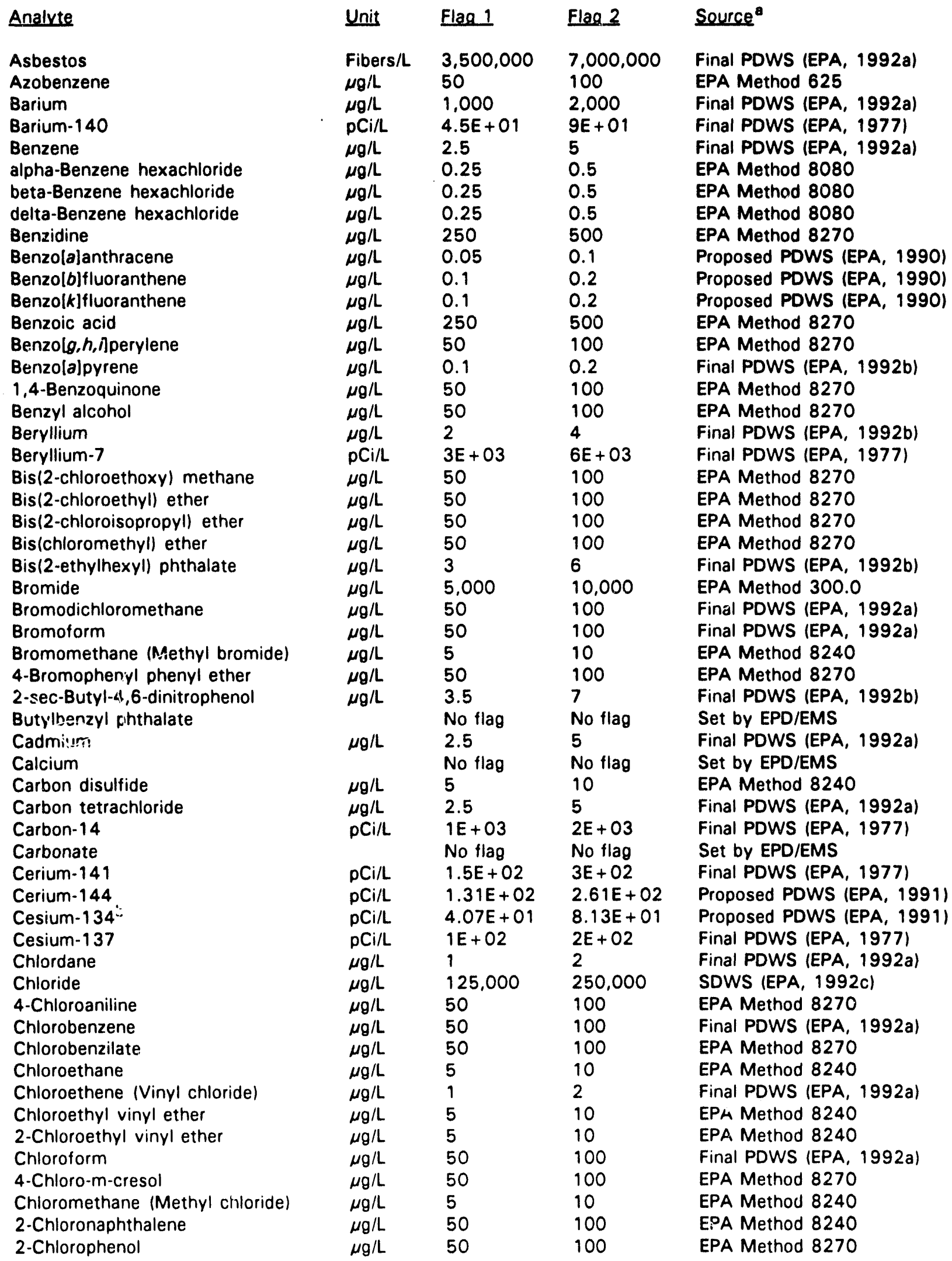




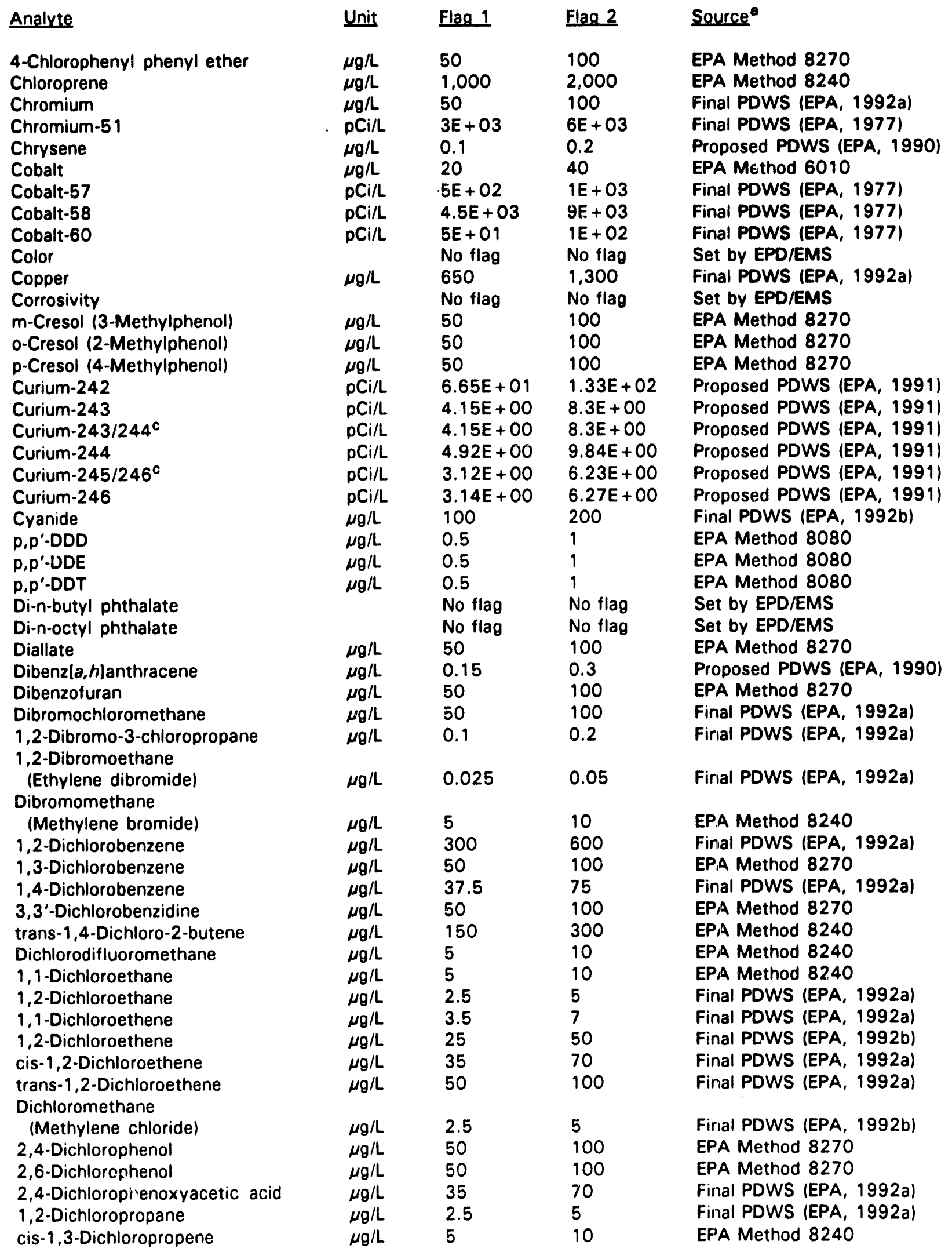




\begin{tabular}{|c|c|c|c|c|}
\hline Analyte & Unit & Flag 1 & Flag 2 & Source $^{\circ}$ \\
\hline trans-1,3-Dichloropropene & $\mu g / L$ & 5 & 10 & EPA Method 8240 \\
\hline Dieldrin & $\mu g / L$ & 2.5 & 5 & EPA Method 8080 \\
\hline Diethyl phthalate & & No flag & No flag & Set by EPD/EMS \\
\hline Dimethoate & $\mu g / L$ & 50 & 100 & EPA Method 8270 \\
\hline p-Dimethylaminoazobenzene & $\mu g / L$ & 50 & 100 & EPA Method 8270 \\
\hline p-(Dimethylamino)ethylbenzene & $\mu g / L$ & 50 & 100 & EPA Method 8270 \\
\hline 7,12-Dimethylbenzla]anthracene & $\mu g / L$ & 50 & 100 & EPA Method 8270 \\
\hline 3,3'-Dimethylbenzidine & $\mu g / L$ & 50 & 100 & EPA Method 8270 \\
\hline a,a-Dimethylphenethylamine & $\mu g / L$ & 50 & 100 & EPA Method 8270 \\
\hline 2,4-Dimethyl phenol & $\mu g / L$ & 50 & 100 & EPA Method 8270 \\
\hline & & No flag & No flag & Set by EPD/EMS \\
\hline $\begin{array}{l}\text { 1,3-Dinitrobenzene } \\
\text { 2,4-Dinitrophenol }\end{array}$ & $\mu g / L$ & 50 & 100 & EPA Method 8270 \\
\hline & $\mu g / L$ & 250 & 500 & EPA Method 8270 \\
\hline & $\mu g / L$ & 50 & 100 & EPA Method 8270 \\
\hline 2,6-Dinitrotoluene & $\mu g / L$ & 50 & 100 & EPA Method 8270 \\
\hline 1,4-Dioxane & $\mu g / L$ & 50 & 100 & EPA Method 8270 \\
\hline Diphenylamine & $\mu g / L$ & 50 & 100 & EPA Method 8270 \\
\hline 1,2-Diphenylhydrazine & $\mu g / L$ & 50 & 100 & EPA Method 8270 \\
\hline Dissolved organic carbon & $\mu g / L$ & 5,000 & 10,000 & EPA Method 9060 \\
\hline Disulfoton & $\mu g / L$ & 50 & 100 & EPA Method 8270 \\
\hline Eh & & No flag & No flag & Set by EPD/EMS \\
\hline alpha-Endosulfan & $\mu g / L$ & 50 & 100 & EPA Method 8270 \\
\hline beta-Endosulfan & $\mu g / L$ & 50 & 100 & EPA Method 8270 \\
\hline Endosulfan I & $\mu g / L$ & 0.5 & 1 & EPA Method 8080 \\
\hline Endosulfan \| & $\mu g / L$ & 0.5 & 1 & EPA Method 8080 \\
\hline Endosulfan sulfate & $\mu g / L$ & 0.5 & 1 & EPA Method 8080 \\
\hline Endrin & $\mu g / L$ & 1 & 2 & Final PDWS (EPA, 1992b) \\
\hline Endrin aldehyde & $\mu g / L$ & 0.5 & 1 & EPA Method 8080 \\
\hline Endrin ketone & & No flag & No flag & Set by EPD/EMS \\
\hline Ethylbenzene & $\mu g / L$ & 350 & 700 & Final PDWS (EPA, 1992a) \\
\hline Ethyl methacrylate & $\mu g / L$ & 50 & 100 & EPA Method 8270 \\
\hline Ethyl methanesulfonate & $\mu g / L$ & 50 & 100 & EPA Method 8270 \\
\hline Europium-152 & $p C i / L$ & $3 E+01$ & $6 E+01$ & Final PDWS (EPA, 1977) \\
\hline Europium-154 & $\mathrm{pCi} / \mathrm{L}$ & $1 E+02$ & $2 E+02$ & Final PDWS (EPA, 1977) \\
\hline Europium-155 & $\mathrm{pCi} / \mathrm{L}$ & $3 E+02$ & $6 E+02$ & Final PDWS (EPA, 1977) \\
\hline Famphur & $\mu g / L$ & 50 & 100 & EPA Method 8270 \\
\hline Fluoranthene & $\mu g / L$ & 50 & 100 & EPA Method 8270 \\
\hline Fluorene & $\mu g / L$ & 50 & 100 & EPA Method 8270 \\
\hline Fluoride & $\mu g / L$ & 2,000 & 4,000 & Final PDWS (EPA, 1992a) \\
\hline Gross alpha & $\mathrm{pCi} / \mathrm{L}$ & $7.5 E+00$ & $1.5 E+01$ & Final PDWS (EPA, 1992a) \\
\hline Heptachlor & $\mu g / L$ & 0.2 & 0.4 & Final PDWS (EPA, 1992a) \\
\hline Heptachlor epoxide & $\mu g / L$ & 0.1 & 0.2 & Final PDWS (EPA, 1992a) \\
\hline Heptachlorodibenzo-p-dioxin & & & & \\
\hline isomers & $\mu g / L$ & 0.00325 & 0.0065 & EPA Method 8280 \\
\hline $1,2,3,4,6,7,8 \cdot H P C D D$ & $\mu g / L$ & 0.00325 & 0.0065 & EPA Method 8280 \\
\hline Heptachlorodibenzo-p-furan & & & & \\
\hline isomers & $\mu g / L$ & 0.00225 & 0.0045 & EPA Method 8280 \\
\hline $1,2,3,4,6,7,8-\mathrm{HPCDF}$ & $\mu g / L$ & 0.00225 & 0.0045 & EPA Method 8280 \\
\hline Hexachlorobenzene & $\mu g / L$ & 0.5 & 1 & Final PDWS (EPA, 1992b) \\
\hline Hexachlorobutadiene & $\mu g / L$ & 50 & 100 & EPA Method 8270 \\
\hline Hexachlorocyclopentadiene & $\mu g / L$ & 25 & 50 & Final PDWS (EPA, 1992b) \\
\hline Hexachlorodibenzo-p-dioxin isomers & $\mu g / L$ & 0.00225 & 0.0045 & EPA Method 8280 \\
\hline $1,2,3,4,7,8-H \times C D D$ & $\mu g / L$ & 0.00225 & 0.0045 & EPA Method 8280 \\
\hline Hexachlorodibenzo-p-furan isomers & $\mu g / L$ & 0.002 & 0.004 & EPA Method 8280 \\
\hline $1,2,3,4,7,8-\mathrm{HXCDF}$ & $\mu \mathrm{g} / \mathrm{L}$ & 0.002 & 0.004 & EPA Method 8280 \\
\hline
\end{tabular}




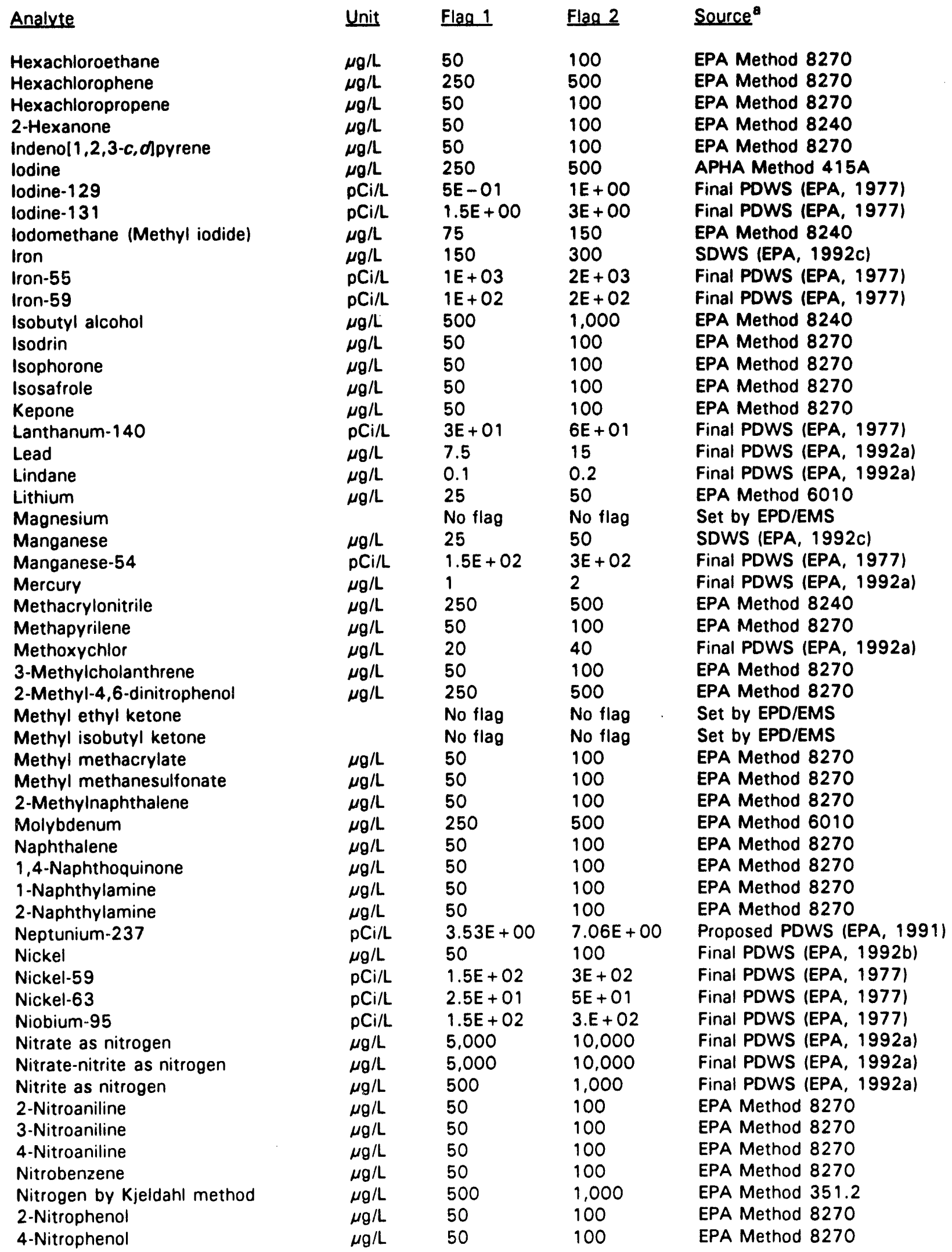




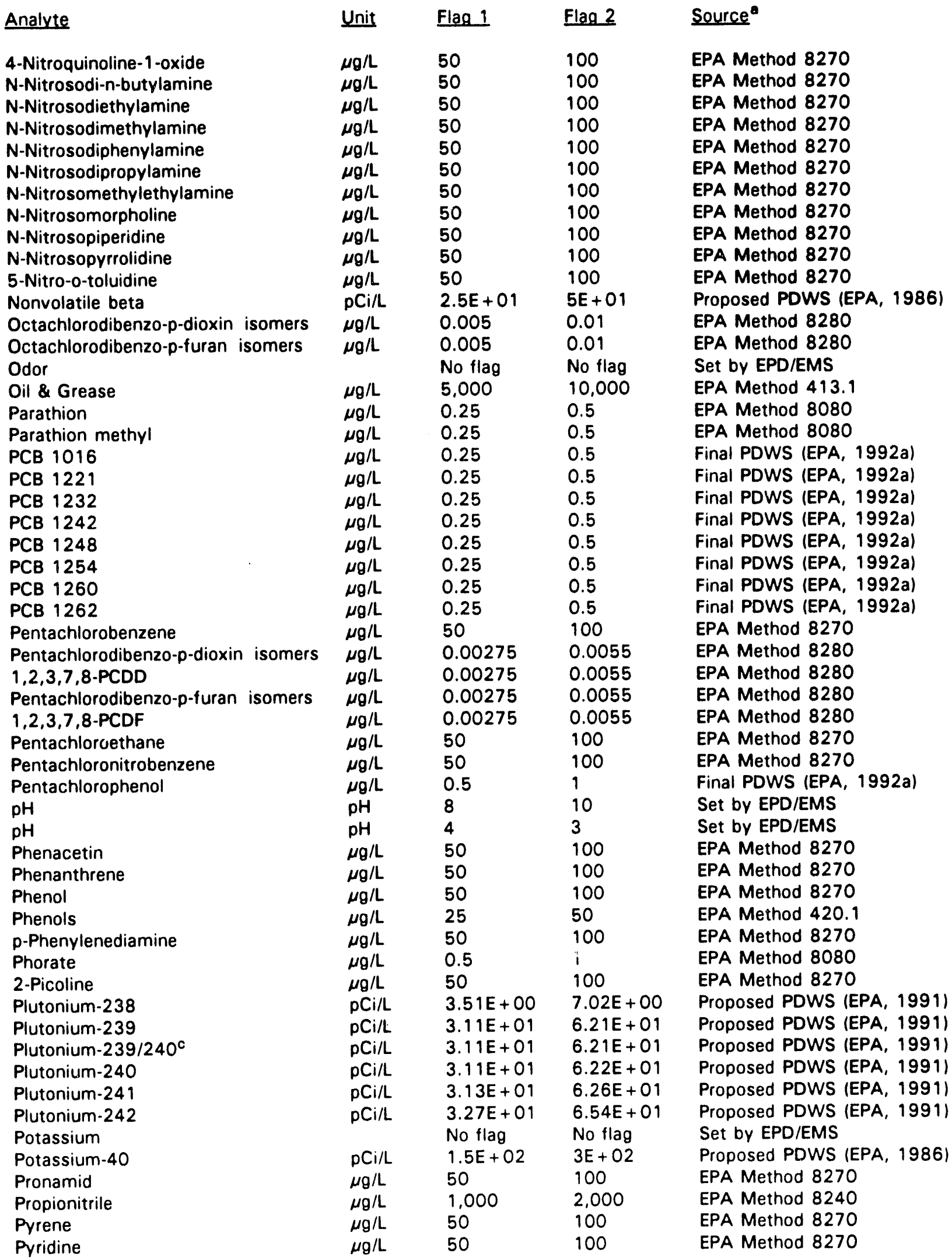


Analyte

Radium (alpha-emitting) ${ }^{d}$

Radium-226

Radium-228

Radon-222

Ruthenium-103

Ruthenium-106

Safrole

Selenium

Silica

Total silica

Silver

Sodium

Sodium-22

Specific conductance

Strontium-89

Strontium-89/90c

Strontium-90

Styrene

Sulfate

Sulfide

Sulfotepp

Surfactants

2,3,7,8-TCDD

$2,3,7,8-$ TCDF

Technetium-99

1,2,4,5-Tetrachlorobenzene

Tetrachlorodibenzo-p-dioxin isomers

Tetrachlorodibenzo-p-furan isomers

1,1,1,2-Tetrachloroethane

1,1,2,2-Tetrachloroethane

Tetrachloroethylene

2,3,4,6-Tetrachlorophenol

Tetraethyl dithiopyrophosphate

Thallium

Thionazin

Thorium-228

Thorium-230

Thorium-232

Thorium-234

Tin

Tin-113

Tolvene

o-Toluidine

Total carbon

Total dissolved solids

Total hydrocarbons

Total inorganic carbon

Total organic carbon

Total organic halogens

Total organic nitrogen

Total petroleum hydrocarbons

Total phosphates (as P)

Total phosphorus

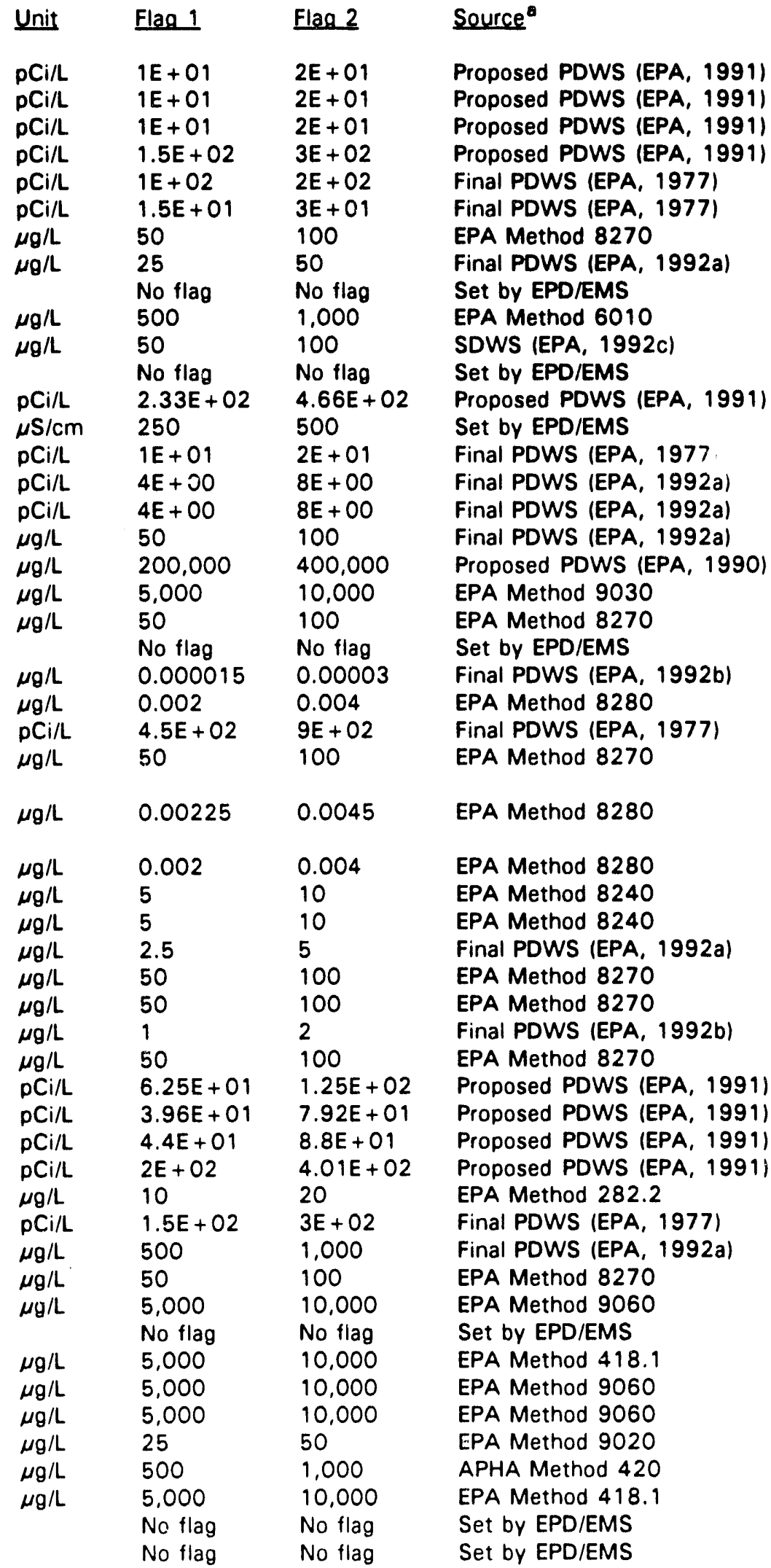




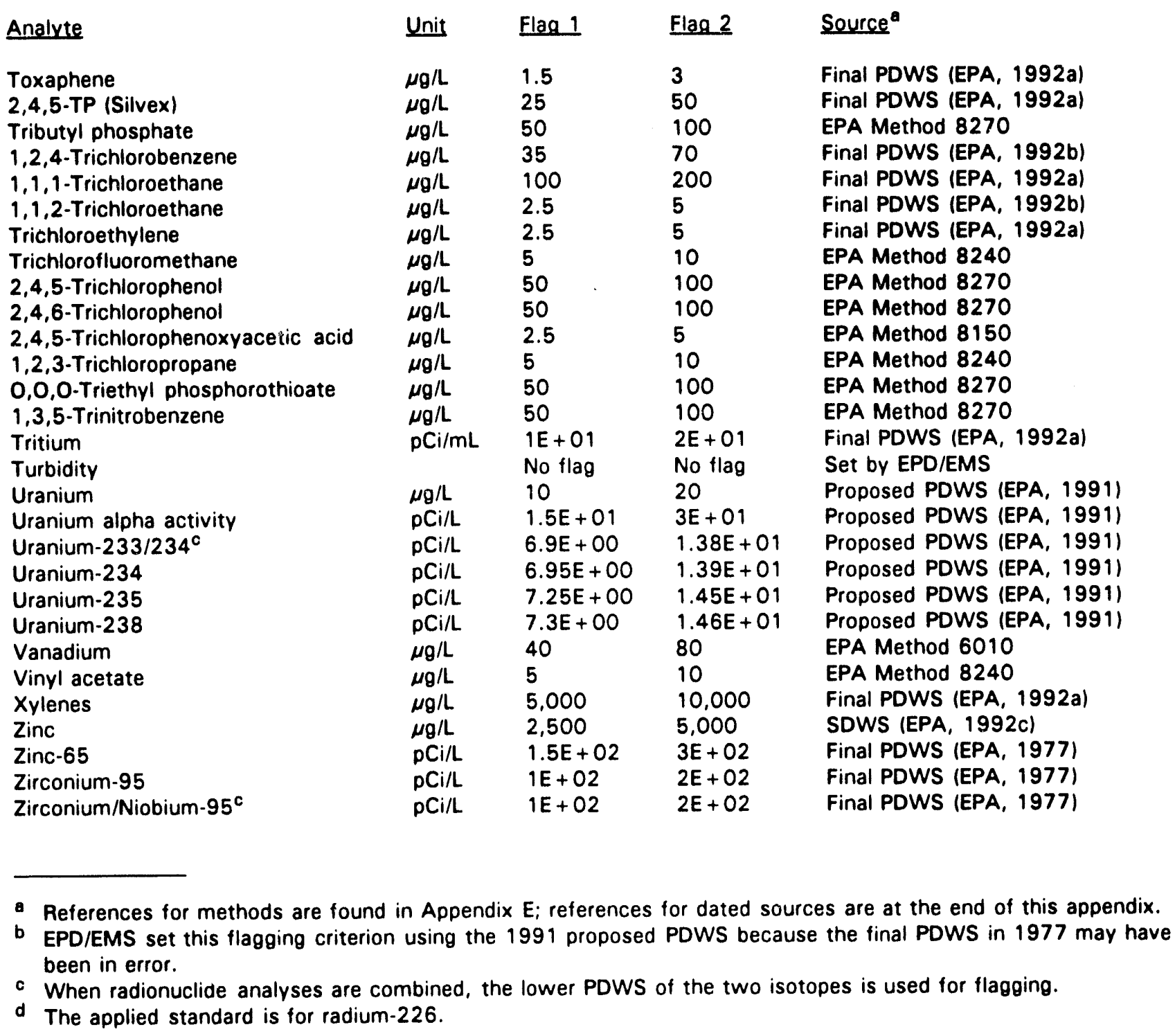

\section{References}

EPA (U.S. Environmental Protection Agencyl, 1977. National Interim Primary Drinking Water Regulations, EPA-570/9-76-003. Washington, DC.

EPA (U.S. Environmental Protection Agency), 1986. Water Pollution Control; National Primary Drinking Water Reguiations, Radionuclides (Proposed). Federal Register, September 30, 1986, pp. 34836-34862. Washington, DC.

EPA (U.S. Environmental Protection Agencyl, 1990. National Primary and Secondary Drinking Water Regulations; Synthetic Organic Chemicals and Inorganic Chemicals (Proposed Rule). Federal Register, July 25, 1990, pp. 30369-30448. Washington, DC.

EPA (U.S. Environmental Protection Agency), 1991. National Primary Drinking Water Regulations; Radionuclides; Proposed Rule. Federal Register, July 18, 1991, pp. 33052-33127. Washington, DC. 
EPA (U.S. Environmental Protection Agency), 1992a. National Primary Drinking Water Regulations, Code of Federal Regulations, Section 40, Part 141, pp. 589-729. Washington, DC.

EPA (U.S. Environmental Protection Agency), 1992b. National Primary Drinking Water Regulations-Synthetic Organic Chemical and Inorganic Chemicals; National Primary Drinking Water Regulations Implementation. Federal Register, July 17, 1992, pp. 31776-31849. Washington, DC.

EPA (U.S. Environmental Protection Agency), 1992c. National Secondary Drinking Water Regulations, Code of Federal Regulations, Section 40, Part 143, pp. 772-776. Washington, DC. 


\section{Appendix C - Figures}




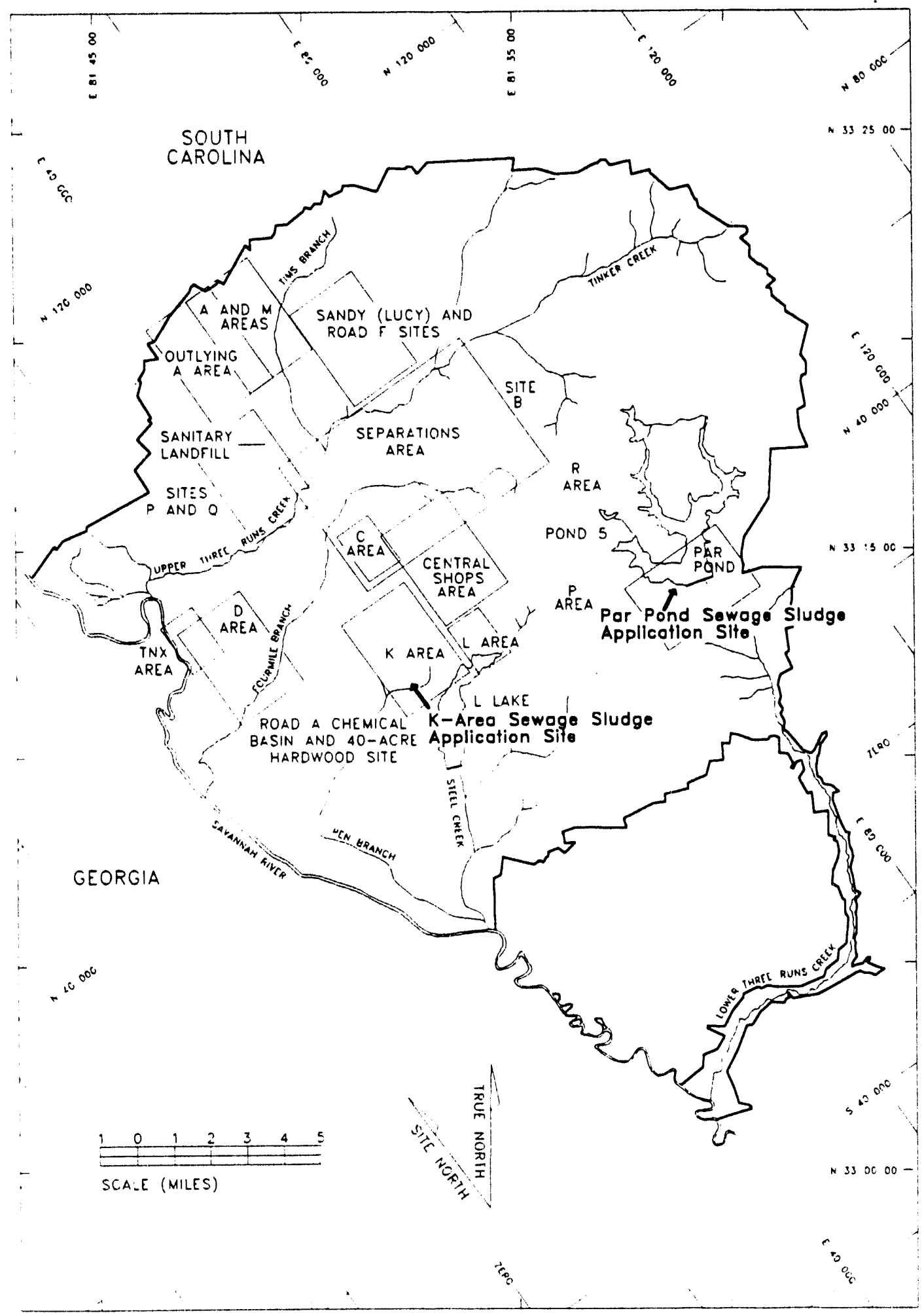

Figure 1. Location of the K·Area and Par Pond Sewage Sludge Application Sites at the Savannah River Site 


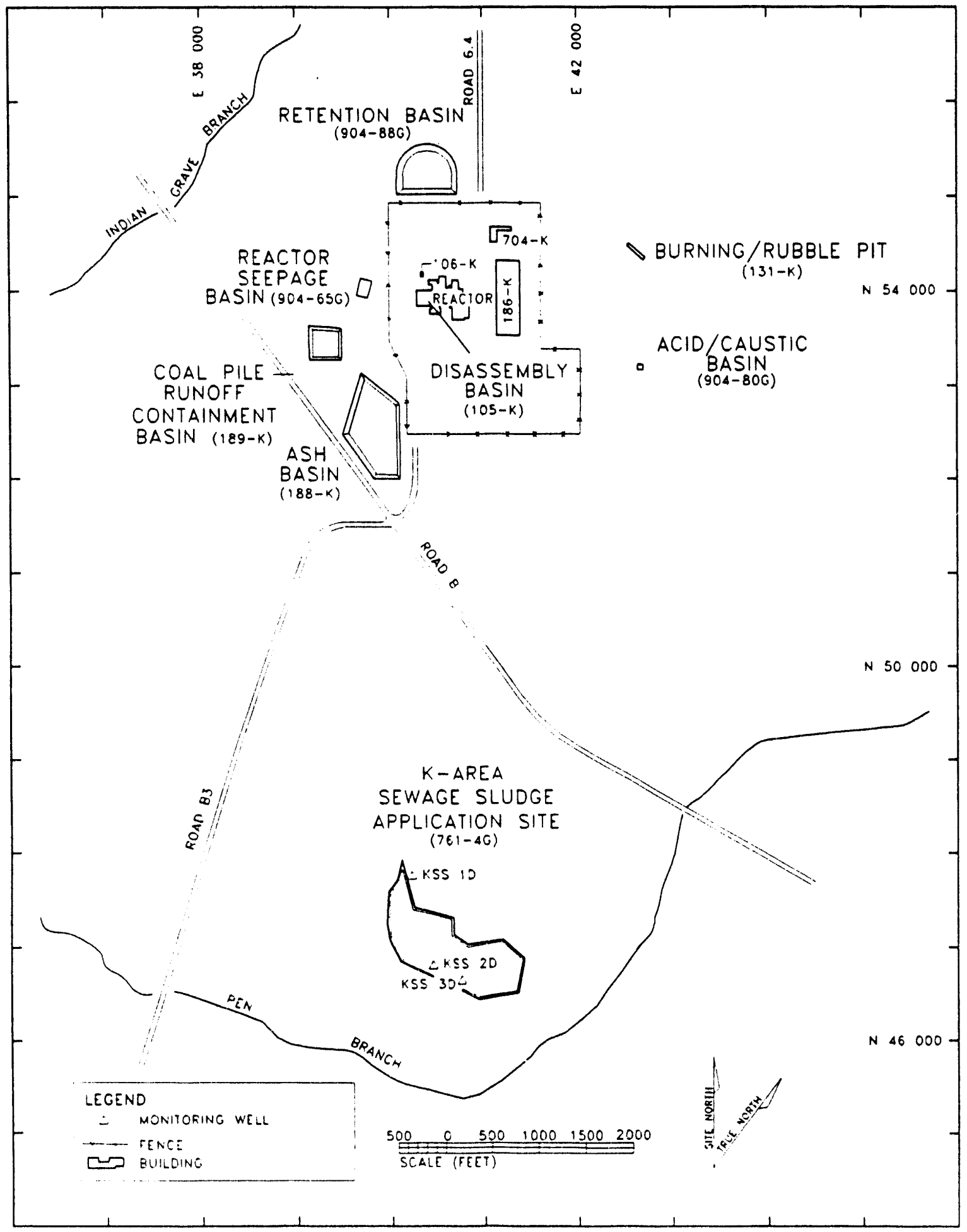

Figure 2. Location of Groundwater Monitoring Wells at the K-Area Sewage Sludge Application Site 


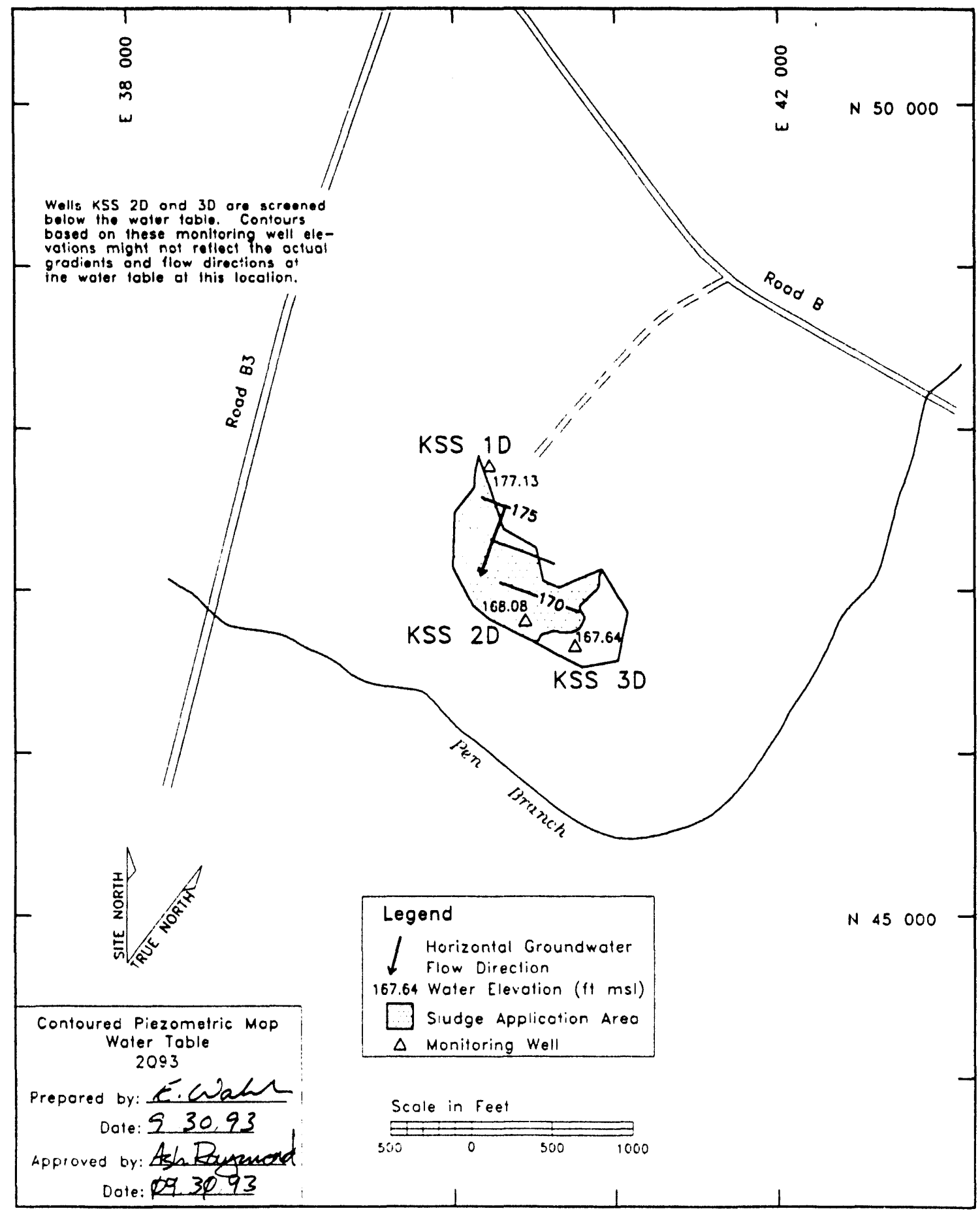

Figure 3. Piezometric Map of the Water Table at the K.Area Sewage Sludge Application Site 


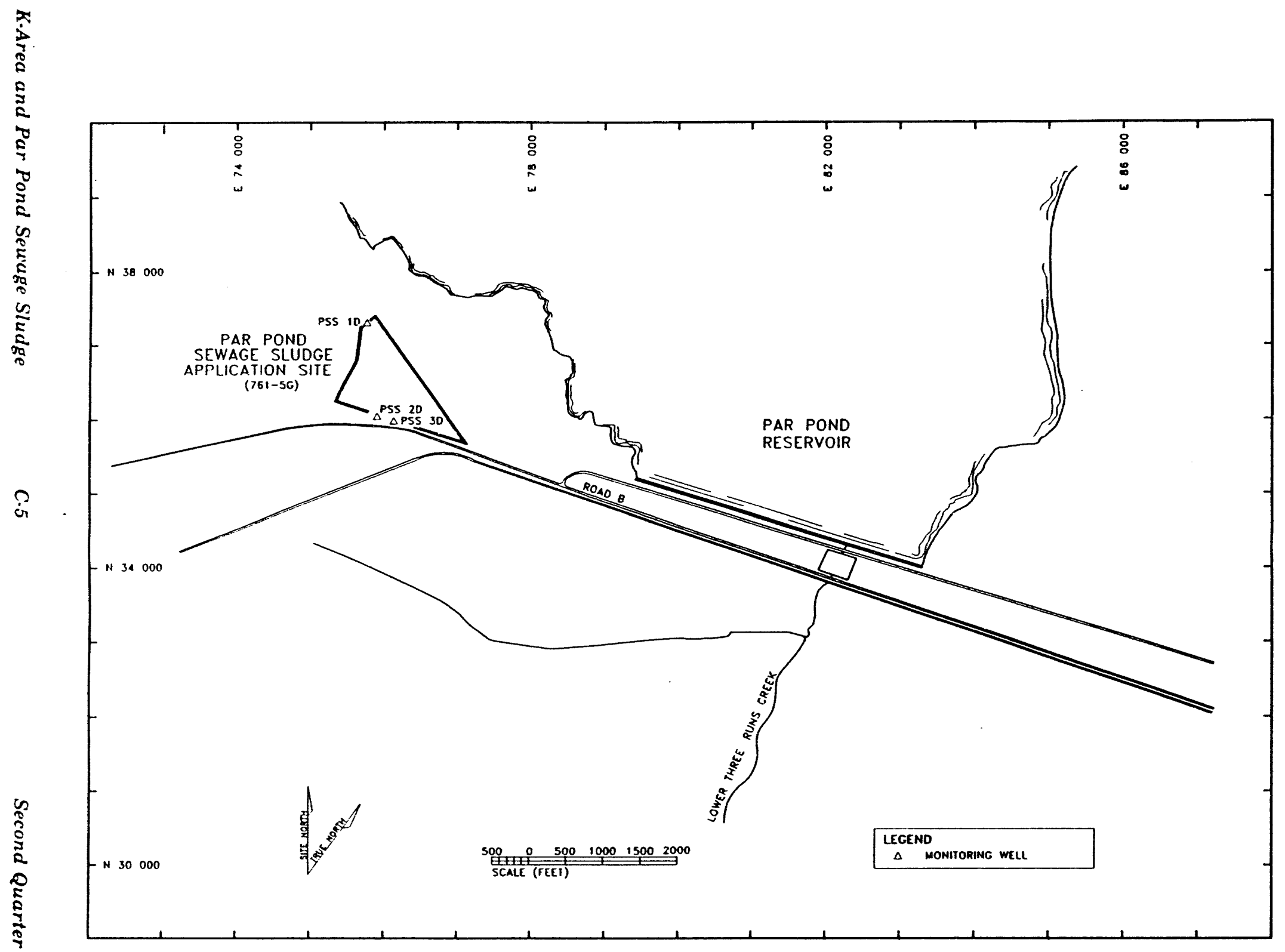

Figure 4. Location of Groundwater Monitoring Wells at the Par Pond Sewage Sludge Application Site 


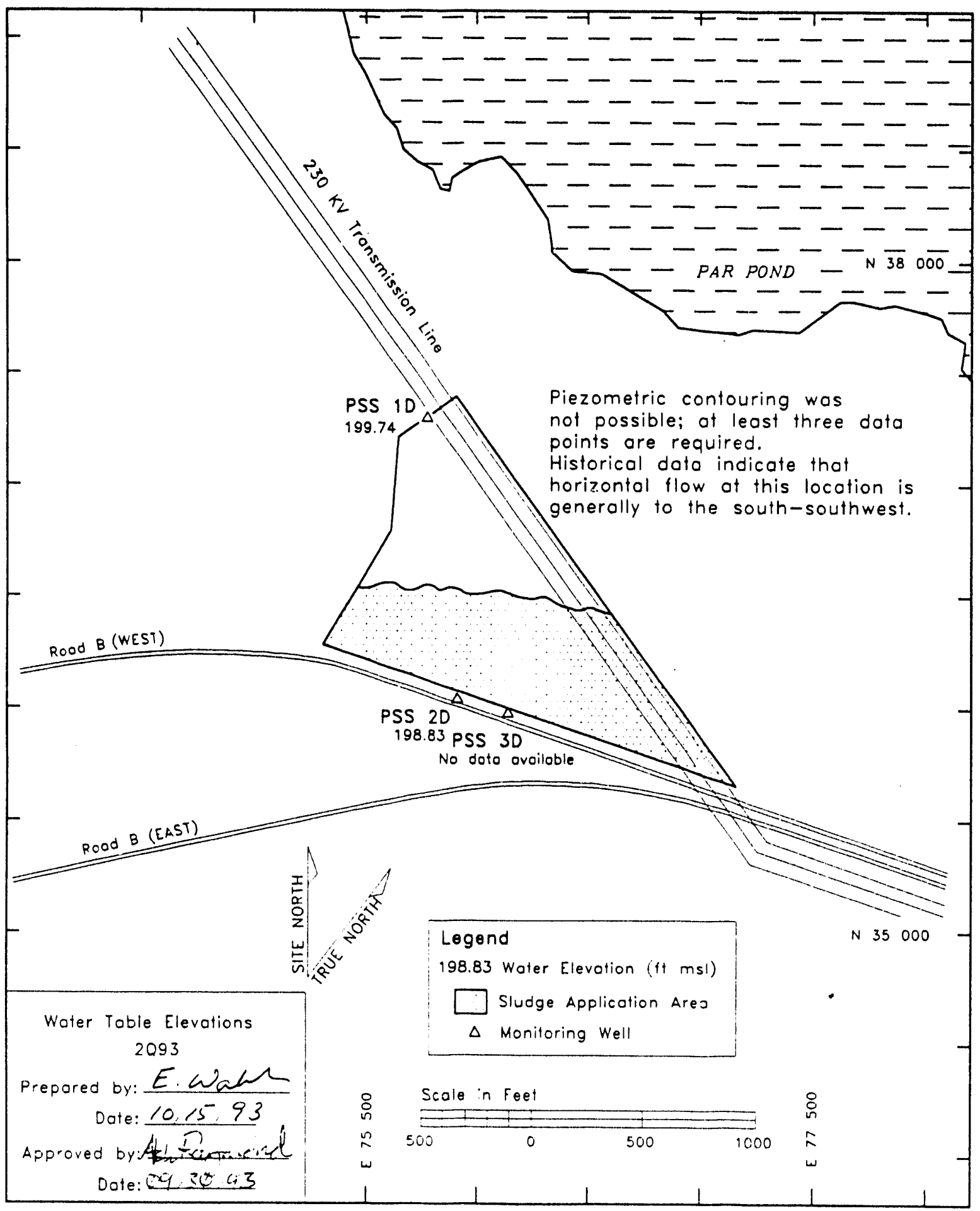

Figure 5. Water Table Elevations at the Par Pond Sewage Sludge Application Site 
WSRC-TR-93-398

Appendix D - Groundwater Monitoring Results Tables 


\section{Key to Reading the Tables}

The following abbreviations may appear in the tabular data:

$B=$ sample collected from well using an open bucket bailer

$\mathrm{BA}=$ Barringer Laboratories, Inc.

$\mathrm{CN}=$ Clemson Technical Center, Inc.

$\mathrm{CS}=$ carbon steel

$\mathrm{D}=$ primary drinking water standard (PDWS)

$E=$ exponential notation (e.g., $1.1 \mathrm{E}-09=1.1 \times 10^{-9}=0.0000000011$ )

EM = Environmental Protection Department/Environmental Monitoring Section (EPD/EMS)

Laboratory

$\mathrm{GE}=$ General Engineering Laboratories

GP = Environmental Physics, Inc.

$\mathrm{H}=$ holding time

1,2,3,4,6,7,8-HPCDD = 1,2,3,4,6,7,8-heptachlorodibenzo-p-dioxin

$1,2,3,4,6,7,8$-HPCDF $=1,2,3,4,6,7,8$-heptachlorodibenzo-p-furan

1,2,3,4,7,8-HXCDD = 1,2,3,4,7,8-hexachlorodibenzo-p-dioxin

$1,2,3,4,7,8$-HXCDF $=1,2,3,4,7,8$-hexachlorodibenzo-p-furan

Lindane = gamma-benzene hexachloride

$\mathrm{mg} / \mathrm{L}=$ milligrams per liter

Mod $=$ modifier

$\mathrm{msl}=$ mean sea level

MSL = million structures per liter

NTU = turbidity unit

$P=$ sample collected from well using a bladder pump

$\mathrm{PCB}=$ polychlorinated biphenyl

1,2,3,7,8-PCDD $=1,2,3,7,8$-pentachlorodibenzo-p-dioxin

1,2,3,7,8-PCDF $=1,2,3,7,8$-pentachlorodibenzo-p-furan

$\mathrm{pCi} / \mathrm{L}=$ picocuries per liter

$\mathrm{pCi} / \mathrm{mL}=$ picocuries per milliliter

PDWS = primar, drinking water standard

$\mathrm{pH}=\mathrm{pH}$ unit

$\mathrm{PVC}=$ polyvinyl chloride

$\mathrm{S}$ = sample collected from well using a single-speed centrifugal downhole pump

Sp. conductance $=$ specific conductance

$\mathrm{SP}=$ Spencer Testing Services, Inc.

TCDD $=$ tetrachlorodibenzo-p-dioxin

TCDF $=$ tetrachlorodibenzo-p-furan

$\mathrm{TM}=\mathrm{TMA} /$ Eberline

$\mathrm{TOC}=$ top of casing

$\mathrm{V}$ = sample collected from well using a variable-speed pump

$\mathrm{WA}=$ Roy F. Weston, Inc.

$\mu \mathrm{g} / \mathrm{L}=$ micrograms per liter

$\mu \mathrm{S} / \mathrm{cm}=$ microsiemens per centimeter 


\section{Holding Times}

Standard analytical methods include a limit, called holding time, on the maximum elapsed time between sample collection and extraction or analysis by the laboratory. In the data tables, a large $\operatorname{dot}(\bullet)$ in the $H$ (holding time) column indicates that holding time was exceeded. Analyses performed beyond holding time may not yield valid results.

The South Carolina Department of Health and Environmental Control allows only 15 minutes to elapse between sampling and analysis for $\mathrm{pH}$. Thus, only field $\mathrm{pH}$ measurements can meet the holding time criterion; laboratory $\mathrm{pH}$ analyses will always exceed it.

The laboratory procedure used for the determination of specific conductance allows one day to elapse between sampling and analysis. Thus, laboratory specific conductance measurements may exceed the holding time criterion.

\section{Data Rounding}

Constituent results in analytical results tables that appear to equal the final PDWS but are not marked in the $D$ (exceeded the final PDWS or screening level) column are below the final PDWS in the database. Values stored in the database contain more significant digits than the reported results. Apparent discrepancies in the tables are due to the rounding of reported results.

\section{Data Qualification}

The contract laboratories continually assess their own accuracy and precision according to U.S. Environmental Protection Agency (EPA) guidelines. They submit sample- or batchspecific quality assurance/quality control information either at the same time as analytical results or in a quarterly summary. Properly defined and used result modifiers (also referred to as qualifiers) can be a key component in assessing data useability. Result modifiers designed by Environmental Protection Department/Environmental Monitoring Section and provided to the primary laboratories are defined below. These modifiers appear in the data tables under the column "Mod." The lettered modifiers are based on EPA's STORET codes.

\section{Result modifier Definition}

(Blank)

Data are not qualified. Number should be interpreted exactly as reported.

A

$J$

L

M
Value reported is the mean of two or more determinations.

Value is estimated because quantitation in the sample or in associated quality control samples did not meet specifications.

Value is off-scale high. The actual value is not known but is known to be greater than the value shown.

Presence of the analyte is verified but not quantified. 
Result modifier

R

T

V

Y

1

2

3

4

6
Definition

Result was rejected because performance requirements in the sample analysis or associated quality control analyses were not met.

Analyte was not detected; if present, it was below the criteria for detection.

Analyte was detected in an associated method blank.

Result was obtained from an unpreserved or improperly preserved sample. Data may not be accurate.

Result may be an underestimation of the true value due to analytical bias.

Result may be an overestimation of the true value due to analytical bias.

The associated result may be of poor precision (high variability) due to analytical bias.

Result is associated with QA results indicating matrix interference.

The associated result is from a reanalysis performed out of holding time due to problems with an earlier analysis. 
Table 1. Maximum Levels of Constituents Exceeding the Final Primary Drinking Water Standards at the K-Area Sewage Sludge Application Site

Well Constituent $\quad$ Unit $\quad \underline{1093} \quad \underline{2093} \quad \underline{\text { Mod }}$ None

Table 2. Maximum Levels of Constituents Exceeding Half the Final Primary Drinking Water Standards or Other Flag 1 or Flag 2 Criteria at the K-Area Sewage Sludge Application Site

$\begin{array}{llll}\text { Well Constituent } & \text { Unit } & 2093 & \text { Mod }\end{array}$

Note: Constituents exceeding half the final PDWS appear italicized. These results do not include field data. 
Table 3. Maximum Levels of Constituents Exceeding the Final Primary Drinking Water Standards at the Par Pond Sewage Sludge Application Site

Well Constituent

Unit

1093

$\underline{2093}$

Mod

None

Table 4. Maximum Levels of Constituents Exceeding Half the Final Primary Drinking Water Standards or Other Flag 1 or Flag 2 Criteria at the Par Pond Sewage Sludge Application Site

Well Constituent

Unit $\underline{2093}$

Mod

Flag

None

Note: Constituents exceeding half the final PDWS appear italicized. These results do not include field data. 
Table 5. Groundwater Monitoring Results for Individual Wells at the K-Area Sewage Sludge Application Site

WELL KSS 1D

$\begin{array}{lllllll}\text { SRS Coord. } & \text { Lat/Longitude } & \text { Screen Zone Elevation } & \text { Top of Casing } & \text { Casing } & \text { Pump } & \text { Formation } \\ \text { N47758.9 } & 33.197023^{\circ} \mathrm{N} & 177.5-157.4 \mathrm{ft} \mathrm{msl} & 229.8 \mathrm{ft} \mathrm{msl} & 4^{\prime \prime} \mathrm{PVC} & \mathrm{S} & \text { Water table } \\ \text { E40219.1 } & 81.653674^{\circ} \mathrm{W} & & & & & \end{array}$

\section{FIELD MEASUREMENTS}

Sample date: $04 / 25 / 93$

Depth to water: $52.67 \mathrm{ft}(16.05 \mathrm{~m})$ below TOC

Water elevation: $177.13 \mathrm{ft}(53.99 \mathrm{~m}) \mathrm{msl}$

Sp. conductance: $88 \mu \mathrm{S} / \mathrm{cm}$

Water evacuated before sampling: $16 \mathrm{gal}$

The well went dry during purging.

\section{LABORATORY ANALYSES}

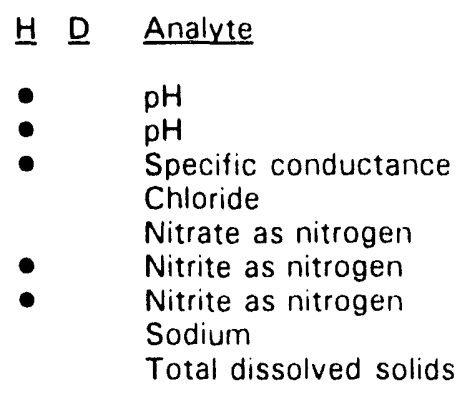

$$
\begin{aligned}
& \text { Result } \\
& \hline 6.2 \\
& 6.2 \\
& 67 \\
& 2,890 \\
& 450 \\
& <10 \\
& <10 \\
& 1,710 \\
& 57,000
\end{aligned}
$$

\begin{tabular}{|c|c|c|c|c|c|c|}
\hline SRS Coord. & Lat/Longitude & Screen Zone Elevation & Top of Casing & Casing & Pump & Formation \\
\hline $\begin{array}{l}N 46803.8 \\
=40437.0\end{array}$ & $\begin{array}{l}33.195266^{\circ} \mathrm{N} \\
81.651247^{\circ} \mathrm{W}\end{array}$ & $164.7-144.6 \mathrm{ft} \mathrm{msl}$ & $192.3 \mathrm{ft} \mathrm{msl}$ & 4" PVC & $S$ & \\
\hline
\end{tabular}

Time: $14: 16$

$\mathrm{pH}: 6.3$

Alkalinity: $35 \mathrm{mg} / \mathrm{L}$

Water temperature: $21.2{ }^{\circ} \mathrm{C}$

Volumes purged: 1.2 well volumes

\section{WELL KSS 2D}

\section{FIELD MEASUREMENTS}

Sample date: $04 / 25 / 93$

Depth to water: $24.22 \mathrm{ft}(7.38 \mathrm{~m})$ below TOC

\begin{tabular}{|c|c|c|c|c|c|c|}
\hline $1 \underline{D}$ & Analyte & Result & Mod & Unit & Flag & $\underline{\text { Lab }}$ \\
\hline & $\mathrm{pH}$ & 5.3 & J & $\mathrm{pH}$ & 0 & WA \\
\hline & Specific conductance & 23 & J & $\mu \mathrm{S} / \mathrm{cm}$ & 0 & $A$ \\
\hline & Chloride & 2,060 & & $\mu \mathrm{g} / \mathrm{L}$ & 0 & WA \\
\hline & Nitrate as nitrogen & 471 & & $\mu \mathrm{g} / \mathrm{L}$ & 0 & WA \\
\hline & Nitrite as nitrogen & 13 & JV & $\mu \mathrm{g} / \mathrm{L}$ & 0 & WA \\
\hline & Sodium & 2,350 & & $\mu \mathrm{g} / \mathrm{L}$ & 0 & WA \\
\hline & Total dissolved solids & 31,000 & V & $\mu \mathrm{g} / \mathrm{L}$ & 0 & W \\
\hline
\end{tabular}

Water elevation: $168.08 \mathrm{ft}(51.23 \mathrm{~m}) \mathrm{msl}$

Sp. conductance: $25 \mu \mathrm{S} / \mathrm{cm}$

Water evacuated before sampling: $82 \mathrm{gal}$
Time: $13: 46$

$\mathrm{pH}: 5.2$

Alkalinity: $3 \mathrm{mg} / \mathrm{L}$

Water temperature: $19.8^{\circ} \mathrm{C}$

Volumes purged: 5.3 well volumes

\section{LABORATORY ANALYSES}

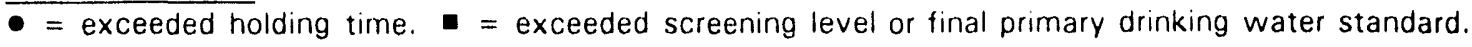


WELL KSS 3D

\begin{tabular}{|c|c|c|c|c|c|c|}
\hline SRS Coord. & Lat/Longitude & Screen Zone Elevation & Top of Casing & Casing & Pume & Formation \\
\hline 44.3 & $33.195420^{\circ} \mathrm{N}$ & $159.3-139.3$ & $185.2 \mathrm{ft} \mathrm{m}$ & PVC & $\mathrm{s}$ & le \\
\hline
\end{tabular}

\section{FIELD MEASUREMENTS}

Sample date: 04/25/93

Depth to water: $17.56 \mathrm{ft}(5.35 \mathrm{~m})$ below TOC

Water elevation: $167.64 \mathrm{ft}(51.10 \mathrm{~m}) \mathrm{msl}$

Sp. conductance: $29 \mu \mathrm{S} / \mathrm{cm}$

Water evacuated before sampling: $99 \mathrm{gal}$

\section{LABORATORY ANALYSES}

H D Analyte

$\mathrm{pH}$

Specific conductance

Chloride

Nitrate as nitrogen

Nitrite as nitrogen

Sodium

Total dissolved solids
Time: 14:06

$\mathrm{pH}: 5.6$

Alkalinity: $7 \mathrm{mg} / \mathrm{L}$

Water temperature: $18.8^{\circ} \mathrm{C}$

Volumes purged: 5.3 well volumes

343

Result

5.7

27

2,160

1,530

127,000
Mod Unit Flag Lab

$J \quad \mathrm{pH} \quad 0 \quad$ WA

$J \mu S / c m$ WA

$\mu \mathrm{g} / \mathrm{L} \quad 0 \quad$ WA

$\mu \mathrm{g} / \mathrm{L} \quad 0 \quad$ WA

$\mu g / L \quad 0 \quad$ WA

$\mu g / L \quad 0 \quad$ WA

$\begin{array}{llll}V & \mu g / L & 0 & \text { WA }\end{array}$

$\overline{- \text { = exceeded holding time. }} \mathbf{a}=$ exceeded screening level or final primary drinking water standard. 


\section{Table 6. Groundwater Monitoring Results for Individual Wells at the Par Pond Sewage Sludge Application Site}

WELL PSS 1D

$\begin{array}{lllllll}\text { SRS Coord. Lat/Longitude } & \text { Screen Zone Elevation } & \text { Top of Casing } & \text { Casing } & & \text { Pump } & \text { Formation } \\ \text { N37298.4 } & 33.231837^{\circ} \mathrm{N} & 202.1 .182 .1 \mathrm{ft} \mathrm{msl} & 219.6 \mathrm{ftmsl} & \text { 4" PVC } & \text { S } & \text { Water table } \\ \text { E75773.3 } & 81.539797^{\circ} \mathrm{W} & & & 202.180\end{array}$

\section{FIELD MEASUREMENTS}

Sample date: $05 / 14 / 93$

Depth to water: $19.86 \mathrm{ft}(6.05 \mathrm{~m})$ below TOC

Water elevation: $199.74 \mathrm{ft}(60.88 \mathrm{~m}) \mathrm{msl}$

Sp. conductance: $16 \mu \mathrm{S} / \mathrm{cm}$

Water evacuated before sampling: 65 gal

\section{LABORATORY ANALYSES}

Time: $11: 28$

pH: 5.1

Alkalinity: $1 \mathrm{mg} / \mathrm{L}$

Water temperature: $20.0^{\circ} \mathrm{C}$

Volumes purged: 5.6 well volumes

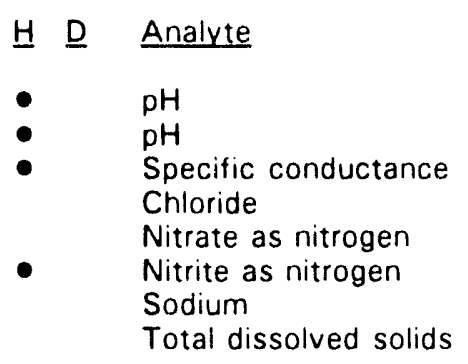

WELL PSS 2D

Result
5.1
5.1
15
1.780
466
$<10$
823
8,000

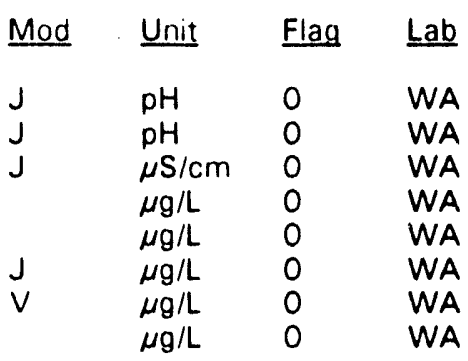

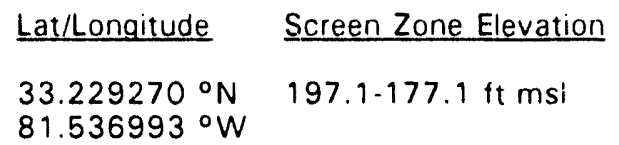

Screen Zone Elevation

197.1-177.1 ft msi

E75910.1

$\begin{array}{lllllll}\text { SRS Coord. Lat/Longitude } & \text { Screen Zone Elevation } & \text { Top of Casing } & \text { Casing } & \text { Pump Formation } \\ \text { N36037.9 } & 33.229270^{\circ} \mathrm{N} & 197.1-177.1 \mathrm{ft} \mathrm{msi} & 228.7 \mathrm{ft} \mathrm{msl} & \text { 4" PVC } & \text { S } & \text { Water table } \\ \text { E75910.1 } & 81.536993^{\circ} \mathrm{W} & & & \end{array}$

\section{FIELD MEASUREMENTS}

Sample date: 05/14/93

Depth to water: $29.87 \mathrm{ft}(9.10 \mathrm{~m})$ below TOC

Water elevation: $198.83 \mathrm{ft}(60.60 \mathrm{~m}) \mathrm{msl}$

Sp. conductance: $22 \mu \mathrm{S} / \mathrm{cm}$

Water evacuated before sampling $116 \mathrm{gal}$

\section{LABORATORY ANALYSES}

Time: 12:05

$\mathrm{pH}: 4.9$

Alkalinity: $0 \mathrm{mg} / \mathrm{L}$

Water temperature: $19.5^{\circ} \mathrm{C}$

Volumes purged: 8.1 well volumes

\section{H $\underline{\text { Analyte }}$ \\ - $\quad$ SH \\ Chloride \\ Nitrate as nitrogen \\ Nitrite as nitrogen \\ Sodium \\ Total dissolved solids}

Result
4.9
20
2,180
992
$<10$
893
20,000

\begin{tabular}{llll} 
Mod & Unit & Flag & Lab \\
\cline { 3 - 4 }$J$ & & & \\
$J$ & $\mu \mathrm{H}$ & 0 & WA \\
& $\mu \mathrm{c} / \mathrm{L}$ & 0 & WA \\
& $\mu \mathrm{g} / \mathrm{L}$ & 0 & WA \\
$\mathrm{J}$ & $\mu \mathrm{g} / \mathrm{L}$ & 0 & WA \\
$\mathrm{V}$ & $\mu \mathrm{g} / \mathrm{L}$ & 0 & WA \\
& $\mu \mathrm{g} / \mathrm{L}$ & 0 & WA
\end{tabular}

$\overline{-}=$ exceeded holding time. $\bullet$ = exceeded screening level or final primary drinking water standard. 
WELL PSS 3D

$\begin{array}{lllllll}\text { SRS Coord. } & \text { Lat/Longitude } & \text { Screen Zone Elevation } & \text { Top of Casing } & \text { Casing } & \text { Pump Formation } \\ \text { N35974.1 } & 33.229501^{\circ} \mathrm{N} & 198.5-178.5 \mathrm{ft} \mathrm{msl} & 234 \mathrm{ft} \mathrm{msl} & 4 \text { " PVC } & \mathrm{S} & \text { Water table } \\ \text { E76138.7 } & 81.5362688^{\circ} \mathrm{W} & & & & \end{array}$

FIELD MEASUREMENTS

Sample date: 05/14/93

Depth to water: Not available

Time: $13: 18$

Water elevation: Not available

$\mathrm{pH}: 4.9$

Alkalinity: $0 \mathrm{mg} / \mathrm{L}$

Sp. conductance: $13 \mu \mathrm{S} / \mathrm{cm}$

Water temperature: $21.2{ }^{\circ} \mathrm{C}$

Water evacuated before sampling: $10 \mathrm{gal}$

The well went dry during purging.

\section{LABORATORY ANALYSES}

\section{H D Analyte}

$\mathrm{pH}$

Specific conductance

Specific conductance

Chloride

Nitrate as nitrogen

Nitrite as nitrogen

Sodium

Total dissolved solids

Result
4.9
11
11
1,800
331
$<10$
592
23,000

Mod

Unit

Flag Lab

$\begin{array}{llll}J & \mathrm{pH} & 0 & \text { WA }\end{array}$

$J \quad \mu S / c m \quad O \quad W A$

$\mu S / \mathrm{cm} \quad 0 \quad$ WA

$\mu \mathrm{g} / \mathrm{L} \quad 0 \quad$ WA

$\mu g / L \quad 0 \quad$ WA

$\mu g / L \quad 0 \quad$ WA

$\mu g / L \quad 0 \quad$ WA

$\mu g / L \quad 0 \quad$ WA

$\overline{-=\text { exceeded holding time. }} \mathbf{-}=$ exceeded screening level or final primary drinking water standard. 


\section{Appendix E - Data Quality/Useability Assessment}




\section{Data Quality/Useability Assessment}

Quality assurance/quality control (QA/QC) procedures relating to accuracy and precision of analyses performed on groundwater samples are followed in the field and laboratory and are reviewed prior to publication of results. The Environmental Protection Department/ Environmental Monitoring Section's (EPD/EMS) review of the volume of analytical data acquired each quarter and presented in various reports is an ongoing process; its review of the QA/QC data cannot be completed in time to meet the deadlines for the reports required by the Resource Conservation and Recovery Act and associated regulations. Other site and regulatory personnel can obtain further information on the data quality and useability in a variety of ways, including those described below.

\section{Data Qualification}

The contract laboratories continually assess their own accuracy and precision according to U.S. Environmental Protection Agency (EPA) guidelines. They submit sample- or batchspecific QA/QC information either at the same time as analytical results or in a quarterly summary. Properly defined and used result inodifiers (also referred to as qualifiers) can be a key component in assessing data useability. Result modifiers designed by EPD/EMS and used by the primary laboratories are presented in Appendix D.

\section{Assessment of Accuracy of the Data}

Accuracy, or the nearness of the reported result to the true concentration of a constituent in a sample, can be assessed in several ways.

A laboratory's general accuracy can be judged by analysis of results obtained from known samples. The non-radionuclide contract laboratories analyze commercial reference samples every quarter at EPD/EMS' request. The results of these analyses are presented in the EPD/EMS quarterly report, The Savannah River Site's Groundwater Monitoring Program. The primary laboratories also seek or maintain state certification by participating periodically in performance studies; reference samples and analysis of results are provided by EPA. Results of these studies also are published in the EPD/EMS quarterly reports.

Analysis of blanks provides a tool for assessing the accuracy of both sampling and laboratory analysis. Results for all field blanks for the quarter can be found in the EPD/EMS quarterly reports. Any field or laboratory blanks that exceeded established minimums are identified in the same reports, in tables associating them with groundwater samples analyzed in the same batches.

Surrogates, organic compounds similar in chemical behavior to the compounds of interest but not normally found in environmental samples, are used to monitor the effect of the matrix on the accuracy of analyses for organic parameters. For example, for analyses of volatile organics by EPA Method 8240 , three surrogate compounds are added to all samples 
and blanks in each analytical batch. In analyses of semivolatile organics, three to four acid compounds and three to four base/neutral compounds are used. Other surrogates are used in pesticides analyses. Percent recoveries for surrogate analyses are calculated by laboratory personnel, reported to EPD/EMS, reviewed, and entered into the database, but they are not published. If recoveries are not within specified limits, the laboratory is expected to re-run the samples or attach result qualifiers to the data identifying the anomalous results.

Sample-specific accuracy for both organic and inorganic parameters can be assessed by examination of matrix spike/matrix spike duplicate results. A sample is analyzed unspiked to determine a baseline set of values. A second portion of sample is spiked with known concentrations of compounds appropriate to the analyses being performed, typically 5 volatile organic compounds for volatile organics analyses, 11 semivolatile compounds for semivolatiles, 6 pesticide compounds for pesticides, all metals for metals analyses, and a known quantity of cyanide for cyanide analysis. The percentage of the spike compound that is recovered (i.e., measured in excess of the value obtained for the unspiked sample) is a direct measure of analytical accuracy. EPA requires matrix spike/matrix spike duplicates to be run at least once per 20 samples of similar matrix.

Matrix spike/matrix spike duplicate results are reported to EPD/EMS but are not published. For organic compounds, according to EPA guidelines, no action is taken on the basis of matrix spike/matrix spike duplicate data alone (i.e., no result modifiers are assigned solely on the basis of matrix spike results); however, the results can indicate if a lab is having a systematic problem in the analysis of one or more analytes.

In the case of inorganic compounds, such as metals, the matrix spike sample analysis provides information about the effect of each sample matrix on the digestion and measurement methodology. Data qualifiers can be assigned on the basis of the percentage of spike recovery and are reported in the published results tables.

\section{Assessment of Precision}

Precision of the analyses, or agreement of a set of replicate results among themselves, is assessed through the use of duplicates (laboratory-initiated) and blind replicates (provided by EPD/EMS). The results of duplicate and replicate analyses are presented in the results tables of the first, second, and third quarter reports. Duplicate and replicate results are not presented in fourth quarter reports; the results tables present instead only the highest result for each analyte for each quarter of the year.

The laboratories assess precision by calculating the relative percent difference, or RPD, for each pair of laboratory-initiated duplicate results. During 1992, at least one of the contract laboratories used a data qualifier (J3) to modify metals analyses when the RPD for laboratory duplicates was greater than $20 \%$.

Additional statistical comparisons of laboratory duplicate and blind replicate results, both intra- and interlaboratory, are presented in the EPD/EMS quarterly reports. The calculation used for these reports is the MRD, or mean relative difference, which is similar to EPA's RPD except that the MRD provides a single value for all of the analyses of a particular com- 
pound, either inter- or intralaboratory, during one quarter. Because detection limits may vary among samples, the MRD requires calculation of a reference detection limit, which is the detection limit at the 90 th percentile of the array of limits in the population of all replicate and duplicate analyses for a given analyte during a particular quarter. The MRD is not method-specific.

\section{Method-Specific Accuracy and Precision}

The contract laboratories' EPA-approved laboratory procedures include QA/QC requirements as an integral part of the methods. Thus, knowledge of the method used in obtaining data is an important component of determining data useability. EPA has conducted extensive research and development on the methods approved for the analysis of water and waste water; information on the accuracy and precision of the method is available from EPA publications, as is full information on required QA/QC procedures. A listing of the methods used by the primary laboratories during first quarter 1992 is given below along with the source for the method description. Many, if not all, of these sources include presentations of representative accuracy and precision results.

Method

EPA120.1

EPA150.1

EPA 160.1

EPA 160.2

EPA 180.1

EPA200.7

EPA206.2

EPA208.2

EPA239.2

EPA245.1

EPA270.2

EPA279.2

EPA300.0

EPA310.1

EPA 325.2

EPA335.3

EPA340.2

EPA353.1

EPA353.2

EPA353.3

EPA354.1

EPA365. 1

EPA365.2

EPA375.4

EPA376.2

APHA403

EPA413.1

APHA4 15A

EPA4 15.1

EPA4 18.1

EPA420.1

EPA420.2

APHA705

\section{Used to Analyze}

Specific conductance

$\mathrm{pH}$

Filterable residue (total dissolved solids)

Nonfilterable residue

Turbidity

Trace elements

Arsenic

Barium

Lead

Mercury

Selenium

Thallium

Inorganics, non-metallics

Alkalinity

Chloride

Cyanide

Fluoride

Nitrogen, nitrate-nitrite

Nitrogen, nitrate, nitrite, or combined

Nitrogen, nitrate-nitrite, or nitrite only

Nitrogen, nitrite

Phosphorus, all forms (reported as total phosphates)

Phosphorus, all forms (reported as total phosphates)

Sulfate, turbidimetric

Sulfide

Alkalinity

Oil \& grease

lodine

Total organic carbon

Petroleum hydrocarbons

Phenolics

Phenolics

Total alpha-emitting radium

\section{Source}

EPA EMSL 1983

EPA EMSL 1983

EPA EMSL 1983

EPA EMSL 1983

EPA EMSL 1983

EPA EMSL 1983

EPA EMSL 1983

EPA EMSL 1983

EPA EMSL 1983

EPA EMSL 1983

EPA EMSL 1983

EPA EMSL 1983

EPA EMSL 1991

EPA EMSL 1983

EPA EMSL 1983

EPA EMSL 1983

EPA EMSL 1983

EPA EMSL 1983

EPA EMSL 1983

EPA EMSL 1983

EPA EMSL 1983

EPA EMSL 1983

EPA EMSL 1983

EPA EMSL 1983

EPA EMSL 1983

APHA 1985

EPA EMSL 1983

APHA 1985

EPA EMSL 1983

EPA EMSL 1983

EPA EMSL 1983

EPA EMSL 1983

APHA 1985 
Method

ASTMD3869C

APHA5320

EPA6010

EPA7041

EPA7060

EPA7421

EPA7470

EPA7740

EPA7841

EPA8010

EPA8020

EPA8080

EPA8140

EPA8150

EPA8240

EPA8270

EPA8280

EPA9012

EPA9020

EPA9030
Used to Analvze

lodide
Dissolved organic halogen
Metals
Antimony
Arsenic
Lead
Mercury
Selenium
Thallium
Halogenated volatile organics
Aromatic volatile organics
Organochlorine pesticides and PCBs
Organophosphorus pesticides
Chlorinated herbicides
GCMS vOA
GCMS semivolatiles
Dioxins and furans
Total cyanide
Total organic halides
Sulfides
Source
ASTM 1992
APHA 1989
EPA 1986
EPA 1986
EPA 1986
EPA 1986
EPA 1986
EPA 1986
EPA 1986
EPA 1986
EPA 1986
EPA 1986
EPA 1986
EPA 1986
EPA 1986
EPA 1986
EPA 1986
EPA 1986
EPA 1986
EPA 1986

An example of the available method-specific QA/QC information is that for the analysis of metals by EPA Method 6010/200.7 (EPA, 1986/EPA EMSL, 1983). The primary laboratories, General Engineering Laboratories (GE) and Roy F. Weston, Inc. (Weston), use this inductively coupled plasma (ICP) atomic emission spectrometric method.

The following precision and accuracy data are based on the experience of seven laboratories that applied the ICP technique to acid-distilled water matrices that had been dosed with various metal concentrates. (Note: not all seven laboratories analyzed all 14 elements.) The references give results for samples having three concentration ranges; the results here are for samples having the lowest values, similar to actual groundwater results for SRS.

ICP Precision and Accuracy Data

\begin{tabular}{llll} 
Element & True value $(\mu \mathrm{g} / \mathrm{L})$ & $\begin{array}{l}\text { Mean reported } \\
\text { value }(\mu \mathrm{g} / \mathrm{L})\end{array}$ & $\begin{array}{l}\text { Mean } \\
\text { ASD }\end{array}$ \\
\cline { 2 - 3 } & 60 & 62 & 33 \\
Alumic & 22 & 19 & 23 \\
Beryllium & 20 & 20 & 9.8 \\
Cadmium & 2.5 & 2.9 & 16 \\
Chromium & 10 & 10 & 18 \\
Cobalt & 20 & 20 & 4.1 \\
Copper & 11 & 11 & 40 \\
Iron & 20 & 19 & 15 \\
Lead & 24 & 30 & 32 \\
Manganese & 15 & 15 & 6.7 \\
Nickel & 30 & 28 & 11 \\
Selenium & 6 & 8.5 & 42
\end{tabular}




\begin{tabular}{|c|c|c|c|}
\hline Element & True value $(\mathrm{mg} / \mathrm{Ll}$ & $\begin{array}{l}\text { Mean reported } \\
\text { value (wg/L) }\end{array}$ & $\begin{array}{l}\text { Mean percent } \\
\text { RSD }^{\mathbf{a}}\end{array}$ \\
\hline $\begin{array}{l}\text { Vanadium } \\
\text { Zinc }\end{array}$ & $\begin{array}{l}70 \\
16\end{array}$ & $\begin{array}{l}69 \\
19\end{array}$ & $\begin{array}{l}2.9 \\
45\end{array}$ \\
\hline
\end{tabular}

Note: In EPA (1986), the column heading is Mean Standard Deviation (\%).

- Relative standard deviation.

As another example, EPA Method 601/8010 (EPA, 1991/EPA, 1986) is used by both GE and Weston for analyses of halogenated volatile organics. In the presentation of the method in both references, the following table gives method-specific accuracy and precision as functions of concentration. Contract laboratories are expected to achieve or at least approach these limits.

Accuracy and Precision as Functions of Concentration for EPA Method 601/8010

\section{Parameter}

Bromodichloromethane

Bromoform

Bromomethane

Carbon tetrachloride

Chlorobenzene

Chloroethane

2-Chloroethyl vinyl etherf

Chloroform

Chloromethane

Dibromochloromethane

1,2-Dichlorobenzene

1,3-Dichlorobenzene

1,4-Dichlorobenzene

1,1-Dichloroethane

1,2-Dichloroethane

1,1-Dichloroethene trans-1,2-Dichloroethene 1,2-Dichloropropane $f$ cis-1,3-Dichloropropene ${ }^{\dagger}$ trans-1,3-Dichloropropene ${ }^{f}$ Methylene chloride

1,1,2,2-Tetrachlorethane

Tetrachloroethylene

1,1,1-Trichloroethane

1,1,2-Trichloroethane

Trichloroethylene

Trichlorofluoromethane

Vinyl chloride

Accuracy as
recovery $x^{\prime a}$
$1.12 C-1.02^{d}$
$0.96 C-2.05$
$0.76 C-1.27$
$0.98 C-1.04$
$1.00 C-1.23$
$0.99 C-1.53$
$1.00 C$
$0.93 C-0.39$
$0.77 C+0.18$
$0.94 C+2.72$
$0.93 C+1.70$
$0.95 C+0.43$
$0.93 C-0.09$
$0.95 C-1.08$
$1.04 C-1.06$
$0.98 C-0.87$
$0.97 C-0.16$
$1.00 C$
$1.00 C$
$1.00 C$
$0.91 C-0.93$
$0.95 C+0.19$
$0.94 C+0.06$
$0.90 C-0.16$
$0.86 C+0.30$
$0.87 C+0.48$
$0.89 C-0.07$
$0.97 C-0.36$

Single analyst precision $(\mu \mathrm{g} / \mathrm{L} .)^{b}$

$0.11 \bar{x}+0.04^{\circ}$

$0.12 \bar{x}+0.58$

$0.28 \bar{x}+0.27$

$0.15 \bar{X}+0.38$

$0.15 \bar{X}-0.02$

$0.14 \bar{X}-0.13$

$0.20 \bar{x}$

$0.13 \bar{x}+0.15$

$0.28 \bar{x}-0.31$

$0.11 \bar{x}+1.10$

$0.20 \bar{x}+0.97$

$0.14 \bar{X}+2.33$

$0.15 \bar{X}+0.29$

$0.09 \bar{x}+0.17$

$0.11 \bar{x}+0.70$

$0.21 \bar{x}-0.23$

$0.11 \bar{x}+1.46$

$0.13 \bar{x}$

$0.18 \bar{x}$

$0.18 \bar{x}$

$0.11 \bar{x}+0.33$

$0.14 \bar{X}+2.41$

$0.14 \bar{X}+0.38$

$0.15 \bar{x}+0.04$

$0.13 \bar{x}-0.14$

$0.13 \bar{x}-0.03$

$0.15 \bar{x}+0.67$

$0.13 \bar{x}+0.65$
Overall precision $(u g / L)^{c}$

$0.20 \bar{x}+1.00$

$0.21 \bar{x}+2.41$

$0.36 \bar{x}+0.94$

$0.20 \bar{x}+0.39$

$0.18 \bar{X}+1.21$

$0.17 \bar{x}+0.63$

$0.35 \bar{x}$

$0.19 \bar{x}-0.02$

$0.52 \bar{x}+1.31$

$0.24 \bar{X}+1.68$

$0.13 \bar{x}+6.13$

$0.26 \bar{x}+2.34$

$0.20 \bar{x}+0.41$

$0.14 \bar{X}+0.94$

$0.15 \bar{X}+0.94$

$0.29 \bar{X}-0.40$

$0.17 \bar{x}+1.46$

$0.23 \bar{X}$

$0.32 \bar{X}$

$0.32 \bar{x}$

$0.21 \bar{x}+1.43$

$0.23 \bar{X}+2.79$

$0.18 \bar{X}+2.21$

$0.20 \bar{X}+0.37$

$0.19 \bar{X}+0.67$

$0.23 \bar{x}+0.30$

$0.26 \bar{x}+0.91$

$0.27 \bar{x}+0.40$

a $X^{\prime}=$ expected recovery for one or more measurements of a sample containing a concentration of $C$, in $\mu g / L$.

b Expected single analyst standard deviation of measurements. 
c Expected interlaboratory standard deviation of measurements.

d $C=$ true value for the concentration, in $\mu g / \mathrm{L}$.

- $\bar{X}=$ average recovery found for measurements of samples containing a concentration of $C$, in $\mu g / L$.

Estimates based on performance in a single laboratory.

\section{References}

APHA (American Public Health Association), 1985. Standard Methods for the Examination of Water and Wastewater, 16th edition. Washington, DC.

APHA (American Public Health Association), 1989. Standard Methods for the Examination of Water and Wastewater, 17th edition. Washington, DC.

ASTM (American Society for Testing and Materials), 1992. 1992 Annual Book of ASTM Standards, Volume 11.02, Water (II). Philadelphia, PA.

EPA (U.S. Environmental Protection Agency), 1986. Test Methods for Evaluating Solid Waste (SW-846), Volumes IA-IC. Washington, DC.

EPA (U.S. Environmental Protection Agency), 1987. Data Quality Objectives for Remedial Response Activities. PB88-131870; EPA/540/G-87/003. Washington, DC.

EPA (U.S. Environmental Protection Agency), 1988a. Contract Laboratory Program Statement of Work for Inorganics Analysis, Multi-Media, Multi-Concentration. SOW No. 788. Washington, DC.

EPA (U.S. Environmental Protection Agency), 1988b. Contract Laboratory Program Statement of Work for Organics Analysis, Multi-Media, Multi-Concentration. SOW No. 288. Washington, DC.

EPA (U.S. Environmental Protection Agency), 1990. Guidance for Data Useability in Risk Assessment. Interim Final. EPA/540/G-90/008. Washington, DC.

EPA (U.S. Environmental Protection Agency), 1991. Guidelines Establishing Test Procedures for the Analysis of Pollutants, Code of Federal Regulations, Title 40, Part 136, Appendix A. Revised July 1, 1991. Washington, DC.

EPA EMSL (U.S. Environmental Protection Agency, Environmental Monitoring and Systems Laboratory), 1979. Handbook for Analytical Quality Control in Water and Wastewater Laboratories. PB-297 451; EPA-600/4-79-019. Cincinnati, $\mathrm{OH}$.

EPA EMSL (U.S. Environmental Protection Agency, Environmental Monitoring and Systems Laboratory), 1983. Methods for Chemical Analysis of Water and Wastes. Revised March 1983. Cincinnati, $\mathrm{OH}$.

EPA EMSL (U.S. Environmental Protection Agency, Environmental Monitoring and Systems Laboratory), 1991. Test Method, The Determination of Inorganic Anions in Water by Ion Chromatography - Method 300.0. Revised August 1991. Cincinnati, OH. 
WSRC-TR-93-398 

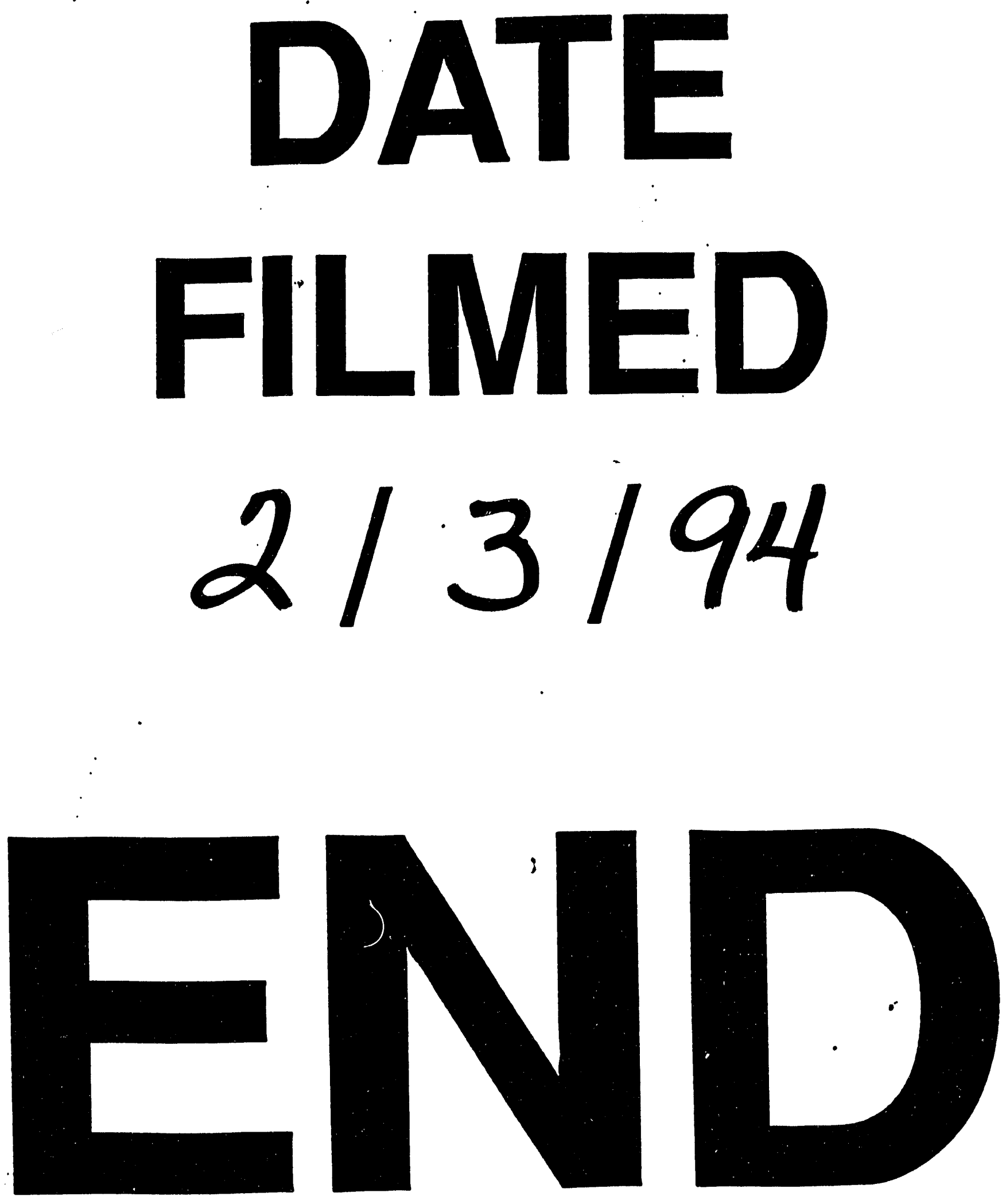
